\title{
On the Passivity-Based Impedance Control of Flexible Joint Robots
}

\author{
Christian Ott, Member, IEEE, Alin Albu-Schäffer, Member, IEEE, Andreas Kugi, Member, IEEE, \\ and Gerd Hirzinger, Fellow, IEEE
}

\begin{abstract}
In this paper, a novel type of impedance controllers for flexible joint robots is proposed. As a target impedance, a desired stiffness and damping are considered without inertia shaping. For this problem, two controllers of different complexity are proposed. Both have a cascaded structure with an inner torque feedback loop and an outer impedance controller. For the torque feedback, a physical interpretation as a scaling of the motor inertia is given, which allows to incorporate the torque feedback into a passivity-based analysis. The outer impedance control law is then designed differently for the two controllers. In the first approach, the stiffness and damping terms and the gravity compensation term are designed separately. This outer control loop uses only the motor position and velocity, but no noncollocated feedback of the joint torques or link side positions. In combination with the physical interpretation of torque feedback, this allows us to give a proof of the asymptotic stability of the closed-loop system based on the passivity properties of the system. The second control law is a refinement of this approach, in which the gravity compensation and the stiffness implementation are designed in a combined way. Thereby, a desired static stiffness relationship is obtained exactly. Additionally, some extensions of the controller to viscoelastic joints and to Cartesian impedance control are given. Finally, some experiments with the German Aerospace Center (DLR) lightweight robots verify the developed controllers and show the efficiency of the proposed control approach.
\end{abstract}

Index Terms-Compliance control, flexible joint robots, impedance control, passivity-based control.

\section{INTRODUCTION}

I MPEDANCE control certainly is one of the core techniques in designing modern robot systems, especially for the growing field of service robotics. The basic control objective of impedance control as formulated in the seminal work of Hogan [1] is the achievement of a desired dynamical relation between external forces and robot movement.

The classical approach to impedance control concentrates on robotic systems in which the joint elasticity is neglected. Consequently, a straightforward application of these techniques

Manuscript received January 29, 2007; revised August 14, 2007. This paper was recommended by Associate Editor P. Rocco and Editor K. Lynch upon evaluation of the reviewers' comments.

C. Ott was with German Aerospace Center (DLR), Institute of Robotics and Mechatronics, Wessling D-82230, Germany. He is now with the Department of Mechano-Informatics, University of Tokyo, Tokyo 113-8656, Japan (e-mail: ott@ynl.t.u-tokyo.ac.jp).

A. Albu-Schäffer and G. Hirzinger are with the German Aerospace Center (DLR), Institute of Robotics and Mechatronics, Wessling D-82230, Germany (e-mail: alin.albu-schaeffer@dlr.de; gerd.hirzinger@dlr.de).

A. Kugi is with the Automation and Control Institute, Vienna University of Technology, Vienna 1040, Austria (e-mail: kugi@acin.tuwien.ac.at).

Color versions of one or more of the figures in this paper are available online at http://ieeexplore.ieee.org.

Digital Object Identifier 10.1109/TRO.2008.915438 to a flexible joint robot usually will not lead to a satisfactory performance. ${ }^{1}$ In fact, the importance of joint elasticity for the design of position and tracking controllers has widely been discussed in the literature [2]-[9].

In this paper, an impedance control law is proposed that is designed for flexible joint robots. The desired impedance is assumed to be a mass-spring-damper system. Furthermore, only the achievement of stiffness and damping is considered herein, while the inertial behavior is left unchanged. In case of a robot with rigid joints, such a stiffness and damping behavior could, in principle, be implemented quite easily with a PD-like controller (formulated in the relevant coordinates). In [10], it was proven that a motor-position-based PD-controller leads to a stable closed-loop system also in case of a robot with flexible joints. Furthermore, in [11], a stability analysis of a hybrid position/force controller for a flexible joint robot without gravitational effects was presented. However, it has been shown that, in practice, often only quite limited performance can be achieved with a restriction to purely motor position (and velocity) based feedback controllers (without additional noncollocated feedback) for the case of a flexible joint robot. In some works, a controller structure based on a feedback of the joint torques as well as the link side positions was considered, and it was shown that this leads to an increase of performance (see, e.g., [12]). This has also already been verified experimentally with the DLR lightweight robots [13]. From a theoretical point of view, this approach is usually justified (for sufficiently high joint stiffness values) by an approximate analysis based on the singular perturbation theory. The feedback of the joint torques is therein considered as the control action of a fast inner control loop that receives its set point values from an outer impedance controller. Furthermore, an integral manifold approach for designing force and impedance controllers for flexible joint robots was presented in [14].

In [15] and [16], a controller with a complete static state feedback (position and torque as well as their first derivatives) was introduced, for which (analogously to [10]) asymptotic stability was shown based on the passivity properties of the controller. In contrast to the classical PD-controller, the motor inertia and the joint stiffness are included in the same passive block as the state feedback controller so that an effective damping of the joint oscillations could be achieved.

In the present paper, a physical interpretation of the torque feedback is given, which allows to include the inner loop torque

\footnotetext{
${ }^{1}$ In terms of damping out the oscillations due to the flexibility in the joint as well as absolute positioning accuracy.
} 


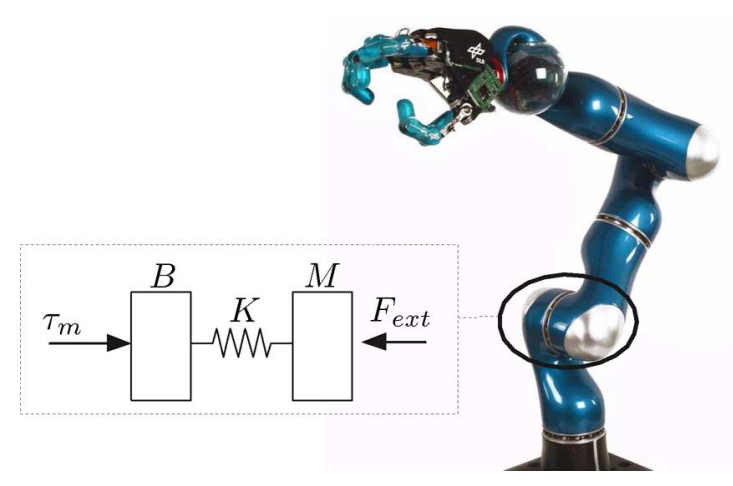

Fig. 1. Sketch of the model for a flexible joint robot.

controller into a passivity-based analysis of the complete closedloop system. It is important to notice that the controller being presented is itself not passive due to the feedback of the joint torque, but it will be shown that the controlled motor dynamics in combination with the torque feedback are passive. Together with the passive (link side) rigid body dynamics, the closed-loop system can, therefore, be represented as a feedback interconnection of passive subsystems.

Furthermore, in [10] and [15], a gravity compensation term based on the desired configuration was used. In case of an impedance controller, this is not appropriate due to the possibly large deviations from the desired configuration which may occur here in case of a low desired stiffness. In this paper, a gravity compensation term will be designed that is based on the measurement of the motor position and is better suited for the use in connection with impedance control. The problem of gravity compensation for flexible joint robots in case of impedance control was also addressed in some recent papers [17], [18]. However, in contrast to our approach, the gravity compensation term in [17] and [18] led to additional lower bounds on the admissible desired stiffness.

Since the controller uses an inner torque feedback loop, a measurement of the joint torques is needed for the implementation. This can be achieved either directly by a joint torque sensor or indirectly by an additional measurement of the link side position. The DLR lightweight robots [19], [20] (see Fig. 1) are equipped with joint torque sensors in order to enable fine manipulation and to enhance the performance when the robot is in interaction with the environment. Therefore, they are ideally suited for the implementation of the presented controllers.

This paper is organized as follows. In Section II, the design idea is described based on a simplified one-dimensional model. The generalization of the design idea to the complete model of a flexible joint robot is then presented in Section III. Some details on the gravity model are given in Section IV. In Section V, an impedance controller based on a separate design of stiffness implementation and gravity compensation is presented. Based on the line of argumentation of the gravity compensation design, an improved controller, which realizes the desired stiffness relation exactly, is presented in Section VI. For the sake of simplicity, the complete controller design and analysis is treated in joint coordinates. The solution, however, is constructed in such a way that the extension to the Cartesian impedance control problem

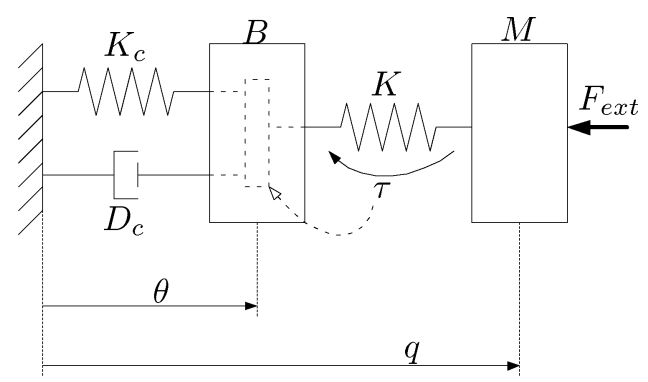

Fig. 2. Motor-position-based PD-control of a single joint. Using torque feedback, the effective motor inertia is scaled down (dashed line).

is rather straightforward. Section VII is devoted to some further extensions of the controller, namely the case of viscoelastic joints and the generalization to Cartesian impedance control. Finally, Sections VIII and IX contain experimental results and conclusions, respectively.

\section{DESIGN IDEA}

In this section, the basic idea of the proposed controller design method is described. It is motivated by some simple considerations for a one-dimensional model.

Consider at first the model of a single flexible joint as it is sketched in Fig. 1 for the second joint of the DLR-LightweightRobot-III. The motor torque $\tau_{m}$ acts here on the rotor inertia $B$ of the motor. ${ }^{2}$ The elasticity of the transmission between the rotor and the following link ${ }^{3}$ of the robot is modeled in form of a linear spring with stiffness $K$.

The goal of the impedance controller is to achieve a desired dynamical behavior with respect to an external force $F_{\text {ext }}$ acting on the link side. In the following, it is assumed that this dynamical behavior is given by a differential equation of second order representing a mass-spring-damper system with mass $M$, desired stiffness $K_{\theta}$, and desired damping $D_{\theta}$. For a robot with rigid joints, this behavior could be realized by a simple PDcontroller with proportional and derivative controller gains set to $K_{c}=K_{\theta}$ and $D_{c}=D_{\theta}$, respectively. For a robot with elastic joints instead, no control law can force the (fourth order) closedloop behavior exactly into such a second-order impedance, since for every joint, four state variables (motor angle $\theta$, link side angle $q$, as well as their first derivatives) are present. If one uses a motor-position-based PD-controller in case of a robot with elastic joints, as shown in Fig. 2 for the one-dimensional case, then the resulting dynamics will also be clearly influenced by the joint elasticity and the motor inertia. Intuitively speaking, the deviation from the desired behavior will be less significant when the rotor inertia $B$ becomes smaller and the joint stiffness $K$ becomes larger.

At this point, it should be mentioned that the joint stiffness values of a typical flexible joint robot are indeed quite large $e^{4}$ but cannot be considered as infinite, and thus, elasticity is not

\footnotetext{
${ }^{2}$ The current controlled motors are modeled as ideal torque sources since the dynamics of the electrical drives are negligible.

${ }^{3}$ In Fig. 1, it is represented in a simplified form with a constant inertia $M$.

${ }^{4}$ For the lower joints of the DLR lightweight robots, these values lie in the range $10.000-15.000 \mathrm{~N} \cdot \mathrm{m} / \mathrm{rad}$.
} 
negligible. By a negative feedback of the joint torque $\tau$, the apparent inertia (of the rotor) can now be scaled down such that the closed-loop system reacts to external forces $F_{\text {ext }}$ as if the rotor inertia were smaller. By reducing the apparent rotor inertia, the desired dynamical behavior is approximated. This approach will be put in concrete terms in the following section for the model of a flexible joint robot. Furthermore, a method for compensating the static influence of the spring $K$ will also be presented. Notice that the design approach presented in this paper thus does not allow to implement a general second-order impedance with arbitrary inertia, but refers to impedance relations with unchanged link side inertia. Its robustness properties due to the passivity-based design make the controller suitable especially for autonomous manipulation tasks in contact with unknown environments. However, for application fields like teleoperation or haptics, the restriction to an unchanged link side inertia may of course be more troublesome.

\section{EFFECTS OF TORQUE FEEDBACK ON THE FLEXIBLE JOINT MODEL}

In this paper, the so-called reduced flexible joint robot model is assumed as proposed by Spong [2]:

$$
\begin{aligned}
\boldsymbol{M}(\boldsymbol{q}) \ddot{\boldsymbol{q}}+\boldsymbol{C}(\boldsymbol{q}, \dot{\boldsymbol{q}}) \dot{\boldsymbol{q}}+\boldsymbol{g}(\boldsymbol{q}) & =\boldsymbol{K}(\boldsymbol{\theta}-\boldsymbol{q})+\boldsymbol{\tau}_{\mathrm{ext}} \\
\boldsymbol{B} \ddot{\boldsymbol{\theta}}+\boldsymbol{K}(\boldsymbol{\theta}-\boldsymbol{q}) & =\boldsymbol{\tau}_{m} .
\end{aligned}
$$

Herein, $\boldsymbol{q} \in \mathbb{R}^{n}$ represents the vector of the $n$ link side joint angles and $\boldsymbol{\theta} \in \mathbb{R}^{n}$ the vector of the corresponding motor angles. The joint torques $\tau \in \mathbb{R}^{n}$ are determined by the linear relationship $\boldsymbol{\tau}=\boldsymbol{K}(\boldsymbol{\theta}-\boldsymbol{q})$, in which $\boldsymbol{K} \in \mathbb{R}^{n \times n}$ is a diagonal matrix containing the individual joint stiffness values $K_{i}$ as diagonal elements, i.e., $\boldsymbol{K}=\operatorname{diag}\left(K_{i}\right)$. The diagonal matrix $\boldsymbol{B} \in \mathbb{R}^{n \times n}$ consists of the rotor inertias $B_{i}$. Furthermore, $\boldsymbol{M}(\boldsymbol{q}) \in \mathbb{R}^{n \times n}$ is the (link side) inertia matrix and $\boldsymbol{C}(\boldsymbol{q}, \dot{\boldsymbol{q}}) \dot{\boldsymbol{q}}$ represents the centrifugal and Coriolis terms of the model. The vector of gravity torques $\boldsymbol{g}(\boldsymbol{q}) \in \mathbb{R}^{n}$ is given by the differential of a potential function $V_{g}(\boldsymbol{q})$, i.e., $\boldsymbol{g}(\boldsymbol{q})=\left(\partial V_{g}(\boldsymbol{q}) / \partial \boldsymbol{q}\right)^{T}$. The motor torques $\boldsymbol{\tau}_{m} \in \mathbb{R}^{n}$ are considered as the control inputs. Finally, the external torques that act on the robot are summarized in the vector $\tau_{\text {ext }} \in \mathbb{R}^{n}$. At this point, moreover, two well-known properties of the robot model shall be mentioned that will be utilized in the following sections.

Property 1: The inertia matrix is symmetric and positive definite:

$$
\boldsymbol{M}(\boldsymbol{q})=\boldsymbol{M}(\boldsymbol{q})^{T}>0 \quad \forall \boldsymbol{q} \in \mathbb{R}^{n} .
$$

Property 2: The matrix $\dot{\boldsymbol{M}}(\boldsymbol{q})-2 \boldsymbol{C}(\boldsymbol{q}, \dot{\boldsymbol{q}})$ fulfills the condition:

$$
\boldsymbol{B} \dot{\boldsymbol{q}}^{T}(\dot{\boldsymbol{M}}(\boldsymbol{q})-2 \boldsymbol{C}(\boldsymbol{q}, \dot{\boldsymbol{q}})) \dot{\boldsymbol{q}}=0 \quad \forall \boldsymbol{q}, \dot{\boldsymbol{q}} \in \mathbb{R}^{n} .
$$

As already described intuitively in the last section, the apparent motor inertia can be reduced from $\boldsymbol{B}$ to $\boldsymbol{B}_{\theta}$ by feeding back the joint torque $\boldsymbol{\tau}=\boldsymbol{K}(\boldsymbol{\theta}-\boldsymbol{q})$. This is realized by the feedback law

$$
\boldsymbol{\tau}_{m}=\boldsymbol{B} \boldsymbol{B}_{\theta}^{-1} \boldsymbol{u}+\left(\boldsymbol{I}-\boldsymbol{B} \boldsymbol{B}_{\theta}^{-1}\right) \boldsymbol{\tau}
$$

where $\boldsymbol{u}$ serves as a new control input. The resulting system dynamics are given by

$$
\begin{aligned}
\boldsymbol{M}(\boldsymbol{q}) \ddot{\boldsymbol{q}}+\boldsymbol{C}(\boldsymbol{q}, \dot{\boldsymbol{q}}) \dot{\boldsymbol{q}}+\boldsymbol{g}(\boldsymbol{q}) & =\boldsymbol{K}(\boldsymbol{\theta}-\boldsymbol{q})+\boldsymbol{\tau}_{\mathrm{ext}} \\
\boldsymbol{B}_{\theta} \ddot{\boldsymbol{\theta}}+\boldsymbol{K}(\boldsymbol{\theta}-\boldsymbol{q}) & =\boldsymbol{u}
\end{aligned}
$$

These equations of motion will be the basis for the design of two joint level impedance control laws. The design in Section $\mathrm{V}$ treats the gravity compensation and the stiffness implementation separately, and is a consequent realization of the design idea described in Section II. But beforehand, some properties of the gravity potential are exposed in the next section.

\section{Properties of the Gravity Potential}

The gravity term $\boldsymbol{g}(\boldsymbol{q})$ corresponds to the differential of the gravity potential $V_{g}(\boldsymbol{q})$, i.e., $\boldsymbol{g}(\boldsymbol{q})=\left(\partial V_{g}(\boldsymbol{q}) / \partial \boldsymbol{q}\right)^{T}$. It is well known that the Hessian $\boldsymbol{H}(\boldsymbol{q}):=\partial^{2} V_{g}(\boldsymbol{q}) / \partial \boldsymbol{q}^{2}$ of the gravity potential has an upper bound if the robot has only rotational joints ${ }^{5}$ [21]. In case the manipulator also instead has prismatic joints, it is useful to consider a subset $\mathcal{Q}^{p}$ of the configuration space $\mathbb{R}^{n}$ in which all the prismatic joints are bounded by their respective workspace boundaries. ${ }^{6}$ In this subset $\mathcal{Q}^{p}$, the existence of an upper bound of the gravity Hessian is guaranteed. From a physical point of view, this bound is a priori not well defined since it clearly depends on the chosen physical units for the translational and rotational coordinates. In order to overcome this problem, particular matrix and vector norms are defined in the following by a scaling with the joint stiffness matrix.

Remark 1: The design of the gravity compensation in Section V-B does not involve the complete dynamics of the manipulator, but refers rather to the static case. Therefore, in this case, the stiffness matrix is the appropriate choice for defining a metric rather than the inertia matrix.

Let $\boldsymbol{R} \in \mathbb{R}^{n \times n}$ be the square root of the joint stiffness matrix ${ }^{7}$ $\boldsymbol{K}$, i.e., $\boldsymbol{K}=\boldsymbol{R}^{T} \boldsymbol{R}$. Then, a vector norm $\|\cdot\|_{K}: \mathbb{R}^{n} \rightarrow \mathbb{R}^{+}$ for a vector $\boldsymbol{v} \in \mathbb{R}^{n}$ can be defined via the Euclidean vector norm $\|\cdot\|_{2}$ as:

$$
\|\boldsymbol{v}\|_{K}:=\|\boldsymbol{R} \boldsymbol{v}\|_{2}=\left(\boldsymbol{v}^{T} \boldsymbol{K} \boldsymbol{v}\right)^{1 / 2} .
$$

The matrix $\boldsymbol{R}$, respectively $\boldsymbol{K}$, is used herein as a normalization of the chosen physical units. Corresponding to this vector norm, the matrix norm $\|\cdot\|_{K}: \mathbb{R}^{n \times n} \rightarrow \mathbb{R}^{+}$for a matrix $\boldsymbol{A} \in \mathbb{R}^{n \times n}$ is defined in the following via the spectral norm ${ }^{8}\|\cdot\|_{i 2}$. In this section, we are interested in the Hessian of the gravity potential. Consequently, it is reasonable to consider the quadratic form $\boldsymbol{v}^{T} \boldsymbol{A} \boldsymbol{v}$ for a matrix $\boldsymbol{A}$. For the vector norm $\|\cdot\|_{K}$ as defined earlier, the following inequality holds:

$$
\left|\boldsymbol{v}^{T} \boldsymbol{A} \boldsymbol{v}\right| \leq\left\|\boldsymbol{R}^{-T} \boldsymbol{A} \boldsymbol{R}^{-1}\right\|_{i 2}\|\boldsymbol{v}\|_{K}^{2} .
$$

\footnotetext{
${ }^{5}$ In this case, the gravity potential can be written as the sum of trigonometric terms of the joint angles.

${ }^{6}$ For a robot with rotational joints, only one has $\mathcal{Q}^{p}=\mathbb{R}^{n}$ instead

${ }^{7}$ Since $\boldsymbol{K}$ is a diagonal matrix, the matrix $\boldsymbol{R}$ is given by $\boldsymbol{R}=\operatorname{diag}\left(\sqrt{K_{i}}\right)$.

${ }^{8}$ The spectral norm is the matrix norm induced by the Euclidean vector norm, and thus, in our case corresponds to the largest eigenvalue.
} 
This motivates the choice

$$
\|\boldsymbol{A}\|_{K}:=\left\|\boldsymbol{R}^{-T} \boldsymbol{A} \boldsymbol{R}^{-1}\right\|_{i 2}
$$

for the definition of the matrix norm $\|\cdot\|_{K}$.

Remark 2: Notice that the term $\boldsymbol{R}^{-T} \boldsymbol{A} \boldsymbol{R}^{-1}$ corresponds to the coordinate transformation of a covariant tensor $\boldsymbol{A}$ of rank two when $\boldsymbol{R}$ is the Jacobian of the coordinate transformation. A linear transformation (i.e., a mixed tensor), instead, would be transformed as $\boldsymbol{R} \boldsymbol{A} \boldsymbol{R}^{-1}$.

Applied to the joint stiffness matrix $\boldsymbol{K}$, this norm clearly gives $\|\boldsymbol{K}\|_{K}=1$. Based on this definition of the matrix norm, one further assumption on the gravity potential is formulated next. This assumption will be useful for the design of the gravity compensation.

Assumption 1: The Hessian $\boldsymbol{H}(\boldsymbol{q}):=\partial^{2} V_{g}(\boldsymbol{q}) / \partial \boldsymbol{q}^{2}$ of the gravity potential $V_{g}(\boldsymbol{q})$ satisfies the condition:

$$
\alpha_{g}:=\sup _{\forall \boldsymbol{q} \in \mathcal{Q}^{p}}\|\boldsymbol{H}(\boldsymbol{q})\|_{K}<\|\boldsymbol{K}\|_{K}=1 .
$$

Notice that this assumption is not restrictive at all. Intuitively speaking, it states nothing else than the fact that the manipulator should be designed properly, in the sense that the joint stiffness is sufficiently high such that, for a fixed motor position, it can prevent the manipulator from falling down under the load of its own weight.

It should also be mentioned that the quantity $\alpha_{g}$ is dimensionless, since it is defined via the norm $\|\cdot\|_{K}$. Also notice that the existence of this bound $\alpha_{g}<1$ implies the following property for the gravity potential that will be useful in the stability analysis in Section V-E.

Property 3: Let $\alpha_{g}$ [as defined in (6)] be an upper bound for the Hessian of the gravity potential $V_{g}(\boldsymbol{q})$ with respect to the $K$-norm. Then, the inequality

$$
\left|V_{g}\left(\boldsymbol{q}_{1}\right)-V_{g}\left(\boldsymbol{q}_{2}\right)+\boldsymbol{g}\left(\boldsymbol{q}_{1}\right)^{T}\left(\boldsymbol{q}_{2}-\boldsymbol{q}_{1}\right)\right| \leq \frac{1}{2} \alpha_{g}\left\|\boldsymbol{q}_{2}-\boldsymbol{q}_{1}\right\|_{K}^{2}
$$

holds for all $\boldsymbol{q}_{1}, \boldsymbol{q}_{2} \in \mathcal{Q}^{p}$.

A proof of this statement can be found in [22].

\section{SeParate Design of Compliance And Gravity COMPENSATION}

In this section, a joint level impedance controller for the model (4)-(5) is proposed. Let the desired impedance at the (constant) virtual equilibrium point $\boldsymbol{q}_{d}$ be specified by a symmetric and positive definite joint stiffness matrix $\boldsymbol{K}_{\theta}$, and a positive definite joint damping matrix $\boldsymbol{D}_{\theta}$. Therefore, the target dynamics of the impedance controller can be written as a mass-spring-damper system of the form

$$
\boldsymbol{M}(\boldsymbol{q}) \ddot{\boldsymbol{q}}+\left(\boldsymbol{C}(\boldsymbol{q}, \dot{\boldsymbol{q}})+\boldsymbol{D}_{\theta}\right) \dot{\boldsymbol{q}}+\boldsymbol{K}_{\theta}\left(\boldsymbol{q}-\boldsymbol{q}_{d}\right)=\boldsymbol{\tau}_{\mathrm{ext}}
$$

in which the link side inertia of the robot is the same as in (1). Consequently, also the corresponding centrifugal and Coriolis terms are present in the target dynamics.

Remark 3: Note that the flexible joint robot model is a $4 n$ dimensional underactuated system in which every joint is represented by four state variables $\left(\theta_{i}, \dot{\theta}_{i}, q_{i}, \dot{q}_{i}\right), i=1, \ldots, n$. There- fore, the desired target dynamics (7) of order $2 n$ can never be achieved exactly by any controller.

Our design approach for approximating this impedance relation follows the ideas described in Section II. The inner loop torque feedback reduces the effect of the motor inertia on the closed-loop dynamics as described in Section III. In addition, we must eliminate the effects of gravity and implement the compliance according to the desired stiffness and damping matrices $\boldsymbol{K}_{\theta}$ and $\boldsymbol{D}_{\theta}$.

The input variable $\boldsymbol{u}$ is thus split up into one term $\boldsymbol{u}_{\text {imp }}$, which actually implements the stiffness and damping, and another term $\boldsymbol{u}_{g}$, which acts as a gravity compensation:

$$
\boldsymbol{u}=\boldsymbol{u}_{\mathrm{imp}}+\boldsymbol{u}_{g} .
$$

\section{A. Implementation of the Compliance Behavior}

According to the design philosophy outlined in Section III, the control input $\boldsymbol{u}_{\mathrm{imp}}$ is simply chosen as a joint space PDcontroller for the motor angles

$$
\boldsymbol{u}_{\mathrm{imp}}=-\boldsymbol{K}_{c}\left(\boldsymbol{\theta}-\boldsymbol{\theta}_{d}\right)-\boldsymbol{D}_{\theta} \dot{\boldsymbol{\theta}}
$$

where the controller gain matrix $\boldsymbol{K}_{c}$ and the virtual equilibrium position on the motor side $\boldsymbol{\theta}_{d}$ are given by:

$$
\begin{aligned}
\boldsymbol{K}_{c} & =\left(\boldsymbol{K}_{\theta}^{-1}-\boldsymbol{K}^{-1}\right)^{-1} \\
\boldsymbol{\theta}_{d} & =\boldsymbol{q}_{d}+\boldsymbol{K}^{-1} \boldsymbol{g}\left(\boldsymbol{q}_{d}\right) .
\end{aligned}
$$

Equation (10) makes allowance for the fact that the controller gain matrix $\boldsymbol{K}_{c}$ acts in series interconnection with the joint spring $\boldsymbol{K}$ (see Fig. 2). The particular form of $\boldsymbol{K}_{c}$ in (10) ensures that in the gravity-free steady state $\left(\boldsymbol{\theta}_{0}, \boldsymbol{q}_{0}\right)$ the demanded stiffness relation $\boldsymbol{\tau}_{\text {ext }}=\boldsymbol{K}_{\theta}\left(\boldsymbol{q}_{0}-\boldsymbol{q}_{d}\right)$ is satisfied exactly.

For the analysis in Section V-D, it is required that not only $\boldsymbol{K}_{\theta}$ but also the controller gain matrix $\boldsymbol{K}_{c}$ is positive definite. Therefore, the following assumption is made, which implies that the controller can implement no joint level stiffness larger than $K$.

Assumption 2: The desired stiffness matrix $\boldsymbol{K}_{\theta}$ is assumed to be symmetric and positive definite, and satisfies the condition $\left(\boldsymbol{K}_{\theta}^{-1}-\boldsymbol{K}^{-1}\right)^{-1}>0$.

So far, the controller (3) and (9) leads to the following closedloop equations:

$$
\begin{aligned}
\boldsymbol{M}(\boldsymbol{q}) \ddot{\boldsymbol{q}}+\boldsymbol{C}(\boldsymbol{q}, \dot{\boldsymbol{q}}) \dot{\boldsymbol{q}}+\boldsymbol{g}(\boldsymbol{q}) & =\boldsymbol{\tau}+\boldsymbol{\tau}_{\mathrm{ext}} \\
\boldsymbol{B}_{\theta} \ddot{\boldsymbol{\theta}}+\boldsymbol{D}_{\theta} \dot{\boldsymbol{\theta}}+\boldsymbol{K}_{c}\left(\boldsymbol{\theta}-\boldsymbol{\theta}_{d}\right)+\boldsymbol{\tau} & =\boldsymbol{u}_{g} .
\end{aligned}
$$

\section{B. Gravity Compensation}

In [10], it has been shown that for a motor-position-based PD-controller, a feedforward term of the gravity torques in the desired steady state $\boldsymbol{q}_{d}$ can be used in order to achieve asymptotic stability. Indeed this leads, for a position controller, usually to good performance because the deviations from the desired position can be kept small. For an impedance controller, however, this is not the case. Here, a pure feedforward action for the gravity compensation does not give satisfactory results because 
large deviations from the virtual equilibrium position may occur in the case of a small desired stiffness $\boldsymbol{K}_{\theta}$. The problem of constructing an online gravity compensation term for a flexible joint robot based solely on the motor position was first treated in [17]. The solution in [17], however, still leads to lower bounds on $\boldsymbol{K}_{\theta}$, limiting the generality of the impedance controller. In contrast to this, the solution presented herein does not require such additional constraints [23], [24].

In the following, a compensation for the static effects of the gravity term $\boldsymbol{g}(\boldsymbol{q})$ is constructed. This compensation is solely based on the motor position and can compensate for the link side gravity torques in a quasi-stationary fashion. Consider first the set $\Omega:=\{(\boldsymbol{q}, \boldsymbol{\theta}) \| \boldsymbol{K}(\boldsymbol{\theta}-\boldsymbol{q})=\boldsymbol{g}(\boldsymbol{q})\}$ of stationary points (for $\tau_{\text {ext }}=\mathbf{0}$ ) for which the torque due to the joint elasticity counterbalances the link side gravity torque. The goal of the gravity compensation is now to construct a compensation term $\overline{\boldsymbol{g}}(\boldsymbol{\theta})$ such that in $\Omega$, the equilibrium condition

$$
\overline{\boldsymbol{g}}(\boldsymbol{\theta})=\boldsymbol{g}(\boldsymbol{q}) \quad \forall(\boldsymbol{q}, \boldsymbol{\theta}) \in \Omega
$$

holds. This means that the gravity compensation term counterbalances the link side gravity torque in all stationary points.

The equation

$$
\boldsymbol{K}(\boldsymbol{\theta}-\boldsymbol{q})=\boldsymbol{g}(\boldsymbol{q})
$$

which describes the set $\Omega$, motivates the definition of a function $\overline{\boldsymbol{q}}(\boldsymbol{\theta})$ that can be understood as a quasi-static estimate of the link side position. Therefore, notice that (15) can obviously be solved uniquely for the motor position $\boldsymbol{\theta}$. Let us denote this solution by:

$$
\boldsymbol{h}_{g}(\boldsymbol{q}):=\boldsymbol{q}+\boldsymbol{K}^{-1} \boldsymbol{g}(\boldsymbol{q}) .
$$

Furthermore, by the use of the contraction mapping theorem (see Proposition 1 given later for more details on this), it can be shown that the inverse function to $\boldsymbol{h}_{g}(\boldsymbol{q})$ exists. Then

$$
\overline{\boldsymbol{q}}(\boldsymbol{\theta}):=\boldsymbol{h}_{g}^{-1}(\boldsymbol{\theta})
$$

which is the solution of (15) for $\boldsymbol{q}$, can be used for the construction of a gravity compensation term of the form:

$$
\boldsymbol{u}_{g}=\overline{\boldsymbol{g}}(\boldsymbol{\theta}):=\boldsymbol{g}(\overline{\boldsymbol{q}}(\boldsymbol{\theta})) .
$$

It is important to notice that, while (14) clearly holds only in $\Omega$, the function $\overline{\boldsymbol{q}}(\boldsymbol{\theta})$ by construction fulfills the equation $\boldsymbol{K}(\boldsymbol{\theta}-$ $\overline{\boldsymbol{q}}(\boldsymbol{\theta}))=\boldsymbol{g}(\overline{\boldsymbol{q}}(\boldsymbol{\theta}))$ for any $\boldsymbol{\theta}$ and independently of $\boldsymbol{q}$.

Finally, the question about the existence of the function $\overline{\boldsymbol{q}}(\boldsymbol{\theta})$ is answered by the following proposition.

Proposition 1: If (6) from Assumption 1 holds globally (i.e., for $\mathcal{Q}^{p}=\mathbb{R}^{n}$ ), the inverse function $\boldsymbol{h}_{g}^{-1}(\boldsymbol{\theta})=\overline{\boldsymbol{q}}(\boldsymbol{\theta})$ of $\boldsymbol{h}_{g}(\boldsymbol{q})=\boldsymbol{q}+\boldsymbol{K}^{-1} \boldsymbol{g}(\boldsymbol{q}): \mathbb{R}^{n} \rightarrow \mathbb{R}^{n}$ exists globally. Moreover, the iteration

$$
\hat{\boldsymbol{q}}_{n+1}=\boldsymbol{T}_{g}\left(\hat{\boldsymbol{q}}_{n}\right)
$$

with $\boldsymbol{T}_{g}(\boldsymbol{q}):=\boldsymbol{\theta}-\boldsymbol{K}^{-1} \boldsymbol{g}(\boldsymbol{q})$ converges for every fixed $\boldsymbol{\theta}$ and for every starting point $\hat{\boldsymbol{q}}_{0}$ to $\overline{\boldsymbol{q}}(\boldsymbol{\theta})$.

Proof: The proposition can be proven by showing first that the mapping $\boldsymbol{T}_{g}(\boldsymbol{q}): \mathbb{R}^{n} \rightarrow \mathbb{R}^{n}$ is a global contraction (see [25]) for the vector norm $\|\cdot\|_{K}$. Since the vector space $\mathbb{R}^{n}$ together with the norm $\|\cdot\|_{K}$ is a Banach space, one must only show that there exists a $\rho<1$, such that $\boldsymbol{T}_{g}(\boldsymbol{q})$ satisfies the condition:

$$
\left\|\boldsymbol{T}_{g}\left(\boldsymbol{q}_{2}\right)-\boldsymbol{T}_{g}\left(\boldsymbol{q}_{1}\right)\right\|_{K} \leq \rho\left\|\boldsymbol{q}_{2}-\boldsymbol{q}_{1}\right\|_{K} \quad \forall \boldsymbol{q}_{1}, \boldsymbol{q}_{2} \in \mathbb{R}^{n}
$$

As shown in [22], this is ensured by (6) from Assumption 1. By the contraction mapping theorem (also called Banach fixed point theorem), one can, therefore, conclude that the mapping $\boldsymbol{T}_{g}(\boldsymbol{q})$ has a unique fixed point $\boldsymbol{q}^{*}=\boldsymbol{T}_{g}\left(\boldsymbol{q}^{*}\right)$ and that the iteration of (19) converges to this fixed point:

$$
\lim _{n \rightarrow \infty} \hat{\boldsymbol{q}}_{n}=\boldsymbol{q}^{*}
$$

By comparing $\boldsymbol{T}_{g}(\boldsymbol{q})$ with $\boldsymbol{h}_{g}(\boldsymbol{q})$, one can easily see that (for each particular value of $\boldsymbol{\theta}$ ) this fixed point $\boldsymbol{q}^{*}$ corresponds to $\overline{\boldsymbol{q}}(\boldsymbol{\theta})$.

While, in general, the inverse function $\boldsymbol{h}_{g}^{-1}(\boldsymbol{\theta})$ cannot be computed directly in practice, it is thus possible to approximate it with arbitrary accuracy by iteration. $>$ From a practical point of view, one or two iteration steps lead to quite satisfactory results in this approximation. Also notice that by a first-order approximation with $\hat{\boldsymbol{q}}_{0}=\boldsymbol{q}_{d}$, one obtains the online gravity compensation term of [17].

In the following analysis, it is therefore assumed that the inverse function $\boldsymbol{h}_{g}^{-1}(\boldsymbol{\theta})$ is known exactly, although it can only be approximated in practice.

Another remark about the range in which Proposition 1 holds is important. The assumption $\mathcal{Q}^{p}=\mathbb{R}^{n}$, which holds, for instance, when the robot has only rotational joints, was needed to ensure that $\boldsymbol{T}_{g}(\boldsymbol{q})$ is a global contraction. If instead $\mathcal{Q}^{p} \subset \mathbb{R}^{n}$, then one must additionally ensure that the points $\hat{\boldsymbol{q}}_{i}$ of the iteration (19) stay in an area, in which $\|\boldsymbol{H}(\boldsymbol{q})\|_{K}<\|\boldsymbol{K}\|_{K}=1$ holds. While this is not a critical issue from a practical point of view, it is difficult to be proven in general.

Since $\overline{\boldsymbol{g}}(\boldsymbol{\theta})$ is the motor torque needed (statically) to prevent the robot from falling down under the action of its own weight, one can see that $\overline{\boldsymbol{g}}(\boldsymbol{\theta})$ must be connected with a potential function $V_{\bar{g}}(\boldsymbol{\theta})$ that is related to the potential energy (gravity plus joint stiffness) of the robot. This potential function will be of interest for the passivity and stability analysis in the next section. A detailed derivation of $V_{\bar{g}}(\boldsymbol{\theta})$ is given in the Appendix. Therein, it is shown that $V_{\bar{g}}(\boldsymbol{\theta})$ can be written as:

$$
\begin{aligned}
V_{\bar{g}}(\boldsymbol{\theta}) & =V_{g}(\overline{\boldsymbol{q}}(\boldsymbol{\theta}))+\frac{1}{2} \boldsymbol{g}(\overline{\boldsymbol{q}}(\boldsymbol{\theta}))^{T} \boldsymbol{K}^{-1} \boldsymbol{g}(\overline{\boldsymbol{q}}(\boldsymbol{\theta})) \\
& =V_{g}(\overline{\boldsymbol{q}}(\boldsymbol{\theta}))+\frac{1}{2}(\overline{\boldsymbol{q}}(\boldsymbol{\theta})-\boldsymbol{\theta})^{T} \boldsymbol{K}(\overline{\boldsymbol{q}}(\boldsymbol{\theta})-\boldsymbol{\theta}) .
\end{aligned}
$$

\section{Controller Formulation}

The complete control law with gravity compensation is summarized as, cf. (3), (8), (9), (18)

$$
\begin{aligned}
\boldsymbol{\tau}_{m} & =\boldsymbol{B} \boldsymbol{B}_{\theta}^{-1} \boldsymbol{u}+\left(\boldsymbol{I}-\boldsymbol{B} \boldsymbol{B}_{\theta}^{-1}\right) \boldsymbol{\tau} \\
\boldsymbol{u} & =-\boldsymbol{K}_{c}\left(\boldsymbol{\theta}-\boldsymbol{\theta}_{d}\right)-\boldsymbol{D}_{\theta} \dot{\boldsymbol{\theta}}+\overline{\boldsymbol{g}}(\boldsymbol{\theta}) .
\end{aligned}
$$




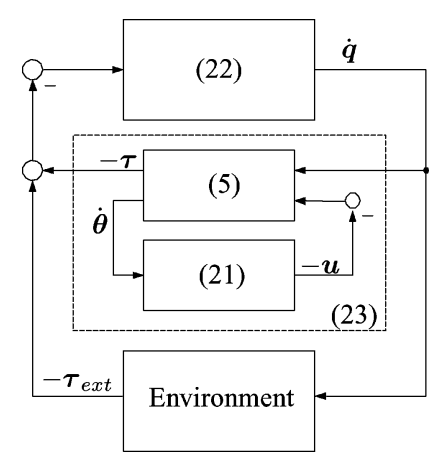

Fig. 3. System representation as an interconnection of passive subsystems.

This leads to the closed-loop system

$$
\begin{aligned}
\boldsymbol{M}(\boldsymbol{q}) \ddot{\boldsymbol{q}}+\boldsymbol{C}(\boldsymbol{q}, \dot{\boldsymbol{q}}) \dot{\boldsymbol{q}}+\boldsymbol{g}(\boldsymbol{q}) & =\boldsymbol{\tau}+\boldsymbol{\tau}_{\mathrm{ext}} \\
\boldsymbol{B}_{\theta} \ddot{\boldsymbol{\theta}}+\boldsymbol{D}_{\theta} \dot{\boldsymbol{\theta}}+\boldsymbol{K}_{c}\left(\boldsymbol{\theta}-\boldsymbol{\theta}_{d}\right)+\boldsymbol{\tau} & =\overline{\boldsymbol{g}}(\boldsymbol{\theta}) .
\end{aligned}
$$

\section{Passivity}

For the passivity analysis, it is assumed that there exists a real $\beta>0$, such that

$$
\left|V_{g}(\boldsymbol{q})\right|<\beta \quad \forall \boldsymbol{q} \in \mathbb{R}^{n}
$$

holds. This is, for instance, satisfied for all robots with rotational joints only (i.e., without prismatic joints). Then also, the gravity torque vector $\boldsymbol{g}(\boldsymbol{q})$ is globally bounded. Furthermore, (24) also implies the boundedness of $V_{\bar{g}}(\boldsymbol{\theta})$ and $\overline{\boldsymbol{g}}(\boldsymbol{\theta})$. Notice that the requirement of a bounded gravity potential is only needed for the passivity analysis, while the proof of the asymptotic stability in Section V-E will also be valid for a general potential.

According to [26] and [27], a sufficient condition for a system (with input $\boldsymbol{u}$ and output $\boldsymbol{y}$ ) to be passive is given by the existence of a continuous storage function $S$, which is bounded from below and for which the derivative with respect to time along the solutions of the system satisfies the inequality $\dot{S} \leq \boldsymbol{y}^{T} \boldsymbol{u}$.

In the following, it will be shown that the system (22)-(23), as outlined in Fig. 3, consists of two passive subsystems in feedback interconnection. Notice that in connection with impedance control, it is often assumed that the environment of the robot can also be described by a passive mapping $\left(\dot{\boldsymbol{q}} \rightarrow-\boldsymbol{\tau}_{\text {ext }}\right)$. The passivity of (22), as a mapping $\left(\boldsymbol{\tau}+\boldsymbol{\tau}_{\text {ext }}\right) \rightarrow \dot{\boldsymbol{q}}$, is well known due to physical reasons and can be shown with the storage function

$$
S_{q}(\boldsymbol{q}, \dot{\boldsymbol{q}})=\frac{1}{2} \dot{\boldsymbol{q}}^{T} \boldsymbol{M}(\boldsymbol{q}) \dot{\boldsymbol{q}}+V_{g}(\boldsymbol{q})
$$

for which (due to Property 2) the derivative along the solutions of (22) is given by

$$
\dot{S}_{q}(\boldsymbol{q}, \dot{\boldsymbol{q}})=\dot{\boldsymbol{q}}^{T}\left(\boldsymbol{\tau}+\boldsymbol{\tau}_{\text {ext }}\right) .
$$

In a similar way, the passivity of (23), as a mapping $\dot{\boldsymbol{q}} \rightarrow-\boldsymbol{\tau}$, can be shown with the storage function:

$$
\begin{aligned}
S_{\theta}(\boldsymbol{q}, \boldsymbol{\theta}, \dot{\boldsymbol{\theta}})= & \frac{1}{2} \dot{\boldsymbol{\theta}}^{T} \boldsymbol{B}_{\theta} \dot{\boldsymbol{\theta}}+\frac{1}{2}(\boldsymbol{\theta}-\boldsymbol{q})^{T} \boldsymbol{K}(\boldsymbol{\theta}-\boldsymbol{q}) \\
& +\frac{1}{2}\left(\boldsymbol{\theta}-\boldsymbol{\theta}_{d}\right)^{T} \boldsymbol{K}_{c}\left(\boldsymbol{\theta}-\boldsymbol{\theta}_{d}\right)-V_{\bar{g}}(\boldsymbol{\theta}) .
\end{aligned}
$$

The derivative of $S_{\theta}(\boldsymbol{q}, \boldsymbol{\theta}, \dot{\boldsymbol{\theta}})$ along the solutions of (23) is then given by

$$
\dot{S}_{\theta}(\boldsymbol{q}, \boldsymbol{\theta}, \dot{\boldsymbol{\theta}})=-\dot{\boldsymbol{\theta}}^{T} \boldsymbol{D}_{\theta} \dot{\boldsymbol{\theta}}-\dot{\boldsymbol{q}}^{T} \boldsymbol{\tau} .
$$

The passivity of the closed-loop system follows directly from (26) and (27) and the fact that the feedback interconnection of passive systems is again passive. It should also be mentioned that these passivity properties are still valid if the PD-controller in (21) is replaced by any other passive (with respect to $\dot{\boldsymbol{\theta}} \rightarrow$ $-\boldsymbol{u}$ ) controller. This structure of a feedback interconnection of passive subsystems, as depicted in Fig. 3, brings along very advantageous robustness properties for the closed-loop system.

\section{E. Stability Analysis}

Next it will be shown that the closed-loop system is asymptotically stable for the case of free motion (i.e., $\boldsymbol{\tau}_{\text {ext }}=\mathbf{0}$ ).

1) Determination of the Steady State: For $\boldsymbol{\tau}_{\text {ext }}=\mathbf{0}$, the steady-state conditions of the system (22)-(23) are given by

$$
\begin{aligned}
\boldsymbol{K}\left(\boldsymbol{\theta}_{0}-\boldsymbol{q}_{0}\right) & =\boldsymbol{g}\left(\boldsymbol{q}_{0}\right) \\
\boldsymbol{K}\left(\boldsymbol{\theta}_{0}-\boldsymbol{q}_{0}\right)+\boldsymbol{K}_{c}\left(\boldsymbol{\theta}_{0}-\boldsymbol{\theta}_{d}\right) & =\overline{\boldsymbol{g}}\left(\boldsymbol{\theta}_{0}\right) .
\end{aligned}
$$

From (14), it follows that

$$
\boldsymbol{K}_{c}\left(\boldsymbol{\theta}_{0}-\boldsymbol{\theta}_{d}\right)=\mathbf{0}
$$

must be satisfied in the steady state. Due to Assumption 2, the matrix $\boldsymbol{K}_{c}$ is positive definite, and hence, the steady state is given by:

$$
\begin{aligned}
& \boldsymbol{\theta}_{0}=\boldsymbol{\theta}_{d} \\
& \boldsymbol{q}_{0}=\boldsymbol{h}_{g}^{-1}\left(\boldsymbol{\theta}_{0}\right)=\boldsymbol{q}_{d} \\
& \dot{\boldsymbol{q}}_{0}=\dot{\boldsymbol{\theta}}_{0}=\mathbf{0} .
\end{aligned}
$$

2) Lyapunov Function: Consider the sum of the storage functions of the subsystems as a Lyapunov function candidate

$$
V(\boldsymbol{q}, \dot{\boldsymbol{q}}, \boldsymbol{\theta}, \dot{\boldsymbol{\theta}})=S_{q}(\boldsymbol{q}, \dot{\boldsymbol{q}})+S_{\theta}(\boldsymbol{q}, \boldsymbol{\theta}, \dot{\boldsymbol{\theta}}) .
$$

First, it is shown that this function is positive definite. Notice that, due to (63) from the Appendix, $V\left(\boldsymbol{q}_{0}, \mathbf{0}, \boldsymbol{\theta}_{0}, \mathbf{0}\right)=$ 0 holds.

By extracting the kinetic part of $V(\boldsymbol{q}, \dot{\boldsymbol{q}}, \boldsymbol{\theta}, \dot{\boldsymbol{\theta}})$

$$
V_{\mathrm{kin}}(\boldsymbol{q}, \dot{\boldsymbol{q}}, \dot{\boldsymbol{\theta}})=\frac{1}{2} \dot{\boldsymbol{q}}^{T} \boldsymbol{M}(\boldsymbol{q}) \dot{\boldsymbol{q}}+\frac{1}{2} \dot{\boldsymbol{\theta}}^{T} \boldsymbol{B}_{\theta} \dot{\boldsymbol{\theta}}
$$

one can see that $V(\boldsymbol{q}, \dot{\boldsymbol{q}}, \boldsymbol{\theta}, \dot{\boldsymbol{\theta}})$ is positive definite with respect to $\dot{\boldsymbol{q}}$ and $\dot{\boldsymbol{\theta}}$ because the inertia matrices are positive definite (Property 1). In order to show that $V(\boldsymbol{q}, \dot{\boldsymbol{q}}, \boldsymbol{\theta}, \dot{\boldsymbol{\theta}})$ is positive definite with respect to the complete state, it is then sufficient to show that the potential part

$$
V_{\mathrm{pot}}(\boldsymbol{q}, \boldsymbol{\theta})=V(\boldsymbol{q}, \dot{\boldsymbol{q}}, \boldsymbol{\theta}, \dot{\boldsymbol{\theta}})-V_{\mathrm{kin}}(\boldsymbol{q}, \dot{\boldsymbol{q}}, \dot{\boldsymbol{\theta}})
$$

is positive definite with respect to $\boldsymbol{q}$ and $\boldsymbol{\theta}$.

Consider first only the part of the potential energy due to $\boldsymbol{K}$. In order to simplify the notation, in the remaining part 
of this section, the function $\overline{\boldsymbol{q}}(\boldsymbol{\theta})$ is written as $\overline{\boldsymbol{q}}$ :

$$
\begin{aligned}
V_{k}(\boldsymbol{q}, \boldsymbol{\theta})= & \frac{1}{2}(\boldsymbol{\theta}-\boldsymbol{q})^{T} \boldsymbol{K}(\boldsymbol{\theta}-\boldsymbol{q}) \\
= & \frac{1}{2}(\boldsymbol{\theta}-\overline{\boldsymbol{q}}+\overline{\boldsymbol{q}}-\boldsymbol{q})^{T} \boldsymbol{K}(\boldsymbol{\theta}-\overline{\boldsymbol{q}}+\overline{\boldsymbol{q}}-\boldsymbol{q}) \\
= & \frac{1}{2} \boldsymbol{g}(\overline{\boldsymbol{q}})^{T} \boldsymbol{K}^{-1} \boldsymbol{g}(\overline{\boldsymbol{q}})+\frac{1}{2}(\overline{\boldsymbol{q}}-\boldsymbol{q})^{T} \boldsymbol{K}(\overline{\boldsymbol{q}}-\boldsymbol{q}) \\
& +(\overline{\boldsymbol{q}}-\boldsymbol{q})^{T} \boldsymbol{g}(\overline{\boldsymbol{q}})
\end{aligned}
$$

Herein, the relationship $\boldsymbol{K}(\boldsymbol{\theta}-\overline{\boldsymbol{q}})=\boldsymbol{g}(\overline{\boldsymbol{q}})$ was used that follows directly from the definition of $\overline{\boldsymbol{q}}(\boldsymbol{\theta})$ in (17). In order to simplify the notation, the deviation of the motor angle from its steady-state value will be denoted by $\tilde{\boldsymbol{\theta}}=$ $\left(\boldsymbol{\theta}-\boldsymbol{\theta}_{d}\right)$ in the following. The potential energy can then be written [with (63) from the Appendix] as follows:

$$
\begin{aligned}
V_{\mathrm{pot}}(\boldsymbol{q}, \boldsymbol{\theta})= & V_{k}(\boldsymbol{q}, \boldsymbol{\theta})+\frac{1}{2} \tilde{\boldsymbol{\theta}}^{T} \boldsymbol{K}_{c} \tilde{\boldsymbol{\theta}}+V_{g}(\boldsymbol{q})-V_{\bar{g}}(\boldsymbol{\theta}) \\
= & V_{k}(\boldsymbol{q}, \boldsymbol{\theta})+\frac{1}{2} \tilde{\boldsymbol{\theta}}^{T} \boldsymbol{K}_{c} \tilde{\boldsymbol{\theta}}+V_{g}(\boldsymbol{q})-V_{g}(\overline{\boldsymbol{q}}) \\
& -\frac{1}{2} \boldsymbol{g}(\overline{\boldsymbol{q}})^{T} \boldsymbol{K}^{-1} \boldsymbol{g}(\overline{\boldsymbol{q}}) .
\end{aligned}
$$

Due to Property 3 , the following inequality holds:

$$
\begin{aligned}
V_{\mathrm{pot}}(\boldsymbol{q}, \boldsymbol{\theta}) \geq & \frac{1}{2}(\overline{\boldsymbol{q}}-\boldsymbol{q})^{T} \boldsymbol{K}(\overline{\boldsymbol{q}}-\boldsymbol{q})+\frac{1}{2} \tilde{\boldsymbol{\theta}}^{T} \boldsymbol{K}_{c} \tilde{\boldsymbol{\theta}} \\
& -\left|V_{g}(\boldsymbol{q})-V_{g}(\overline{\boldsymbol{q}})+(\overline{\boldsymbol{q}}-\boldsymbol{q})^{T} \boldsymbol{g}(\overline{\boldsymbol{q}})\right| \\
\geq & \frac{1}{2}\left(1-\alpha_{g}\right)|| \overline{\boldsymbol{q}}-\left.\boldsymbol{q}\right|_{K} ^{2}+\frac{1}{2} \tilde{\boldsymbol{\theta}}^{T} \boldsymbol{K}_{c} \tilde{\boldsymbol{\theta}}
\end{aligned}
$$

The right-hand side of the last inequality is nonnegative for all $(\boldsymbol{q}, \boldsymbol{\theta}) \in \mathcal{Q}^{p}$, since by Assumption 1, the bound $\alpha_{g}$ satisfies the inequality condition $\alpha_{g}<1$. Therefore, one can conclude that the considered candidate Lyapunov function is positive definite in $\mathcal{Q}^{p}$.

3) Derivative of the Lyapunov Function: The change of $V(\boldsymbol{q}, \dot{\boldsymbol{q}}, \boldsymbol{\theta}, \dot{\boldsymbol{\theta}})$ along the solutions of the system (22)-(23) (for $\boldsymbol{\tau}_{\text {ext }}=\mathbf{0}$ ) is given by:

$$
\dot{V}(\boldsymbol{q}, \dot{\boldsymbol{q}}, \boldsymbol{\theta}, \dot{\boldsymbol{\theta}})=\dot{S}_{q}(\boldsymbol{q}, \dot{\boldsymbol{q}})+\dot{S}_{\theta}(\boldsymbol{q}, \boldsymbol{\theta}, \dot{\boldsymbol{\theta}})=-\dot{\boldsymbol{\theta}}^{T} \boldsymbol{D}_{\theta} \dot{\boldsymbol{\theta}}
$$

Due to the fact that the matrix $\boldsymbol{D}_{\theta}$ is positive definite, it can be concluded that the equilibrium point is stable. Furthermore, asymptotic stability can be shown by the use of the invariance principle of LaSalle [25]. According to this theorem, the system state will converge to the largest positively invariant set for which $\dot{\boldsymbol{\theta}}=\mathbf{0}$ holds. From the system equations, it follows that there does not exist any trajectory for which $\dot{\boldsymbol{\theta}}=\mathbf{0}$ holds for all times $t>0$ except for the restriction to the equilibrium point. Therefore, the following proposition can be concluded.

Proposition 2: Under the Assumptions 1 and 2, the system (22)-(23) is asymptotically stable for the case of free motion (i.e., for $\boldsymbol{\tau}_{\text {ext }}=\mathbf{0}$ ). Moreover, if Assumption 1 holds globally (i.e., for $\mathcal{Q}^{p}=\mathbb{R}^{n}$ ), then the system is even globally asymptotically stable.

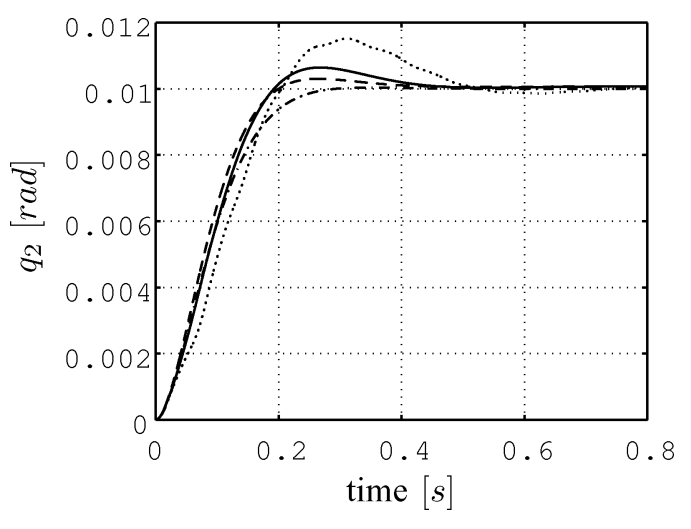

Fig. 4. Simulated joint angle for a step-wise excitation of $10 \mathrm{~N} \cdot \mathrm{m}$ (dashdotted line: desired impedance, dotted line: $\boldsymbol{B}_{\theta}=\boldsymbol{B}$, solid line: $\boldsymbol{B}_{\theta}=\boldsymbol{B} / 3$, dashed line: $\left.\boldsymbol{B}_{\theta}=\boldsymbol{B} / 10\right)$.

\section{F. Controller Discussion}

The passivity analysis in Section V-D shows that the closedloop system can be seen as a feedback interconnection of passive subsystems. In many applications, the environment can also be treated as a passive system with respect to the input $\dot{\boldsymbol{q}}$ and the output $-\boldsymbol{\tau}_{\text {ext }}$. Therefore, one can conclude very advantageous robustness properties of the whole system. Stability is, for instance, also guaranteed for arbitrary errors in the dynamical parameters of the inertia matrices $\boldsymbol{M}(\boldsymbol{q})$ and $\boldsymbol{B}$ as long as these matrices remain positive definite and $\boldsymbol{B}$ remains a diagonal matrix.

Concerning the formulation of the gravity compensation term, it should be mentioned that, in contrast to any related previous works, no lower bounds are imposed on the positive definite matrix $\boldsymbol{K}_{\theta}$ for stability reasons, meaning that the desired stiffness $\boldsymbol{K}_{\theta}$ can be chosen arbitrarily close to zero.

At this point, it is illustrative to evaluate up to which extent the controller approximates the desired impedance behavior from (7). Therefore, a small simulation of the 7 degreesof-freedom DLR-Lightweight-Robot-II ([19], see also Fig. 6 in Section VIII) will be shown. In this simulation, the closed-loop response for a step-wise excitation using an external torque of $10 \mathrm{~N} \cdot \mathrm{m}$ at joint 2 is evaluated. The simulation was performed with different values for $\boldsymbol{B}_{\theta}$ in order to demonstrate the role of the torque feedback in the controller. The desired stiffness and damping matrices are set to diagonal matrices with an overall stiffness of $1000 \mathrm{~N} \cdot \mathrm{m} / \mathrm{rad}$ and the desired damping is set to $\boldsymbol{D}_{\theta}=\operatorname{diag}\{100,100,100,100,1,1,1\}$ corresponding to the different effective inertia for the lower and upper joints. In the following, only the motion of joint 2 , onto which the external force is exerted, will be analyzed in detail. In Fig. 4, the link side joint angle of this axis is shown. First, the dash-dotted line shows the step response of the desired impedance (7). Secondly, the dotted line shows the control action for the controller without any torque feedback, i.e., with $\boldsymbol{B}_{\theta}$ set to $\boldsymbol{B}$. One can see some higher frequency oscillations and also a rather huge overshoot. Next, the same step response is shown with $\boldsymbol{B}_{\theta}=\boldsymbol{B} / 3$ (solid line) and with $\boldsymbol{B}_{\theta}=\boldsymbol{B} / 10$ (dashed line). The former corresponds to a moderate torque feedback while the latter is in the 


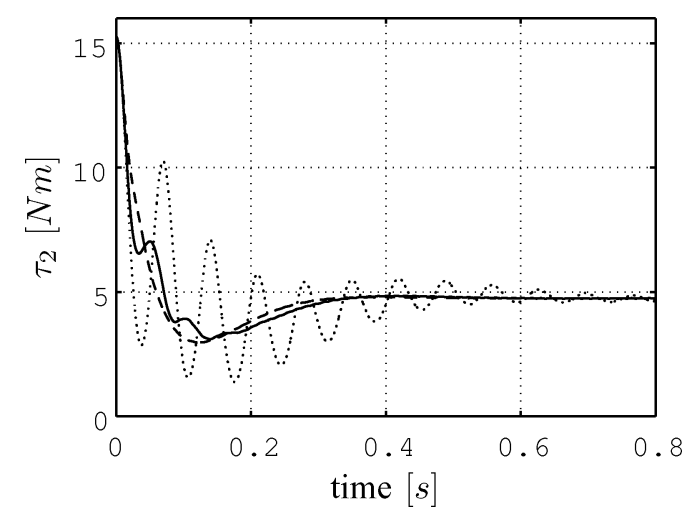

Fig. 5. Simulated joint torque for a step-wise excitation of $10 \mathrm{~N} \cdot \mathrm{m}$ (dotted line: $\boldsymbol{B}_{\theta}=\boldsymbol{B}$, solid line: $\boldsymbol{B}_{\theta}=\boldsymbol{B} / 3$, dashed line: $\boldsymbol{B}_{\theta}=\boldsymbol{B} / 10$ ).

range of the highest gains that could be implemented for this robot, in practice, considering the noise of the torque sensor. One can see that for higher torque feedback gains, the desired dynamics is approximated better. In order to have a closer look at the oscillation damping performance, Fig. 5 shows the simulated joint torque. One can see that the torque oscillations, observed for the case of $\boldsymbol{B}_{\theta}=\boldsymbol{B}$ (dotted line), are already damped out quite effectively by the lower gain $\boldsymbol{B}_{\theta}=\boldsymbol{B} / 3$ (solid line) and cannot be observed any more for the higher gain $\boldsymbol{B}_{\theta}=\boldsymbol{B} / 10$ (dashed line).

The solution presented so far, however, has one disadvantage. The stiffness and damping term $\boldsymbol{u}_{\text {imp }}$ and the gravity compensation term $\boldsymbol{u}_{g}$ were designed separately. While the term $\boldsymbol{u}_{\text {imp }}$ guarantees the correct stiffness relation (statically) for the gravity-free case, the term $\boldsymbol{u}_{g}$ was designed for the case of free motion, i.e., for $\tau_{\text {ext }}=\mathbf{0}$. In the above analysis, it was shown that these two terms can indeed be combined without jeopardizing the passivity and stability of the system. But it is not guaranteed any more that the desired static relation $\boldsymbol{\tau}_{\text {ext }}=\boldsymbol{K}_{\theta}\left(\boldsymbol{q}_{0}-\boldsymbol{q}_{d}\right)$ holds exactly for all $\boldsymbol{\tau}_{\text {ext }} \neq \mathbf{0}$. In fact, a small steady-state error can also be observed for the simulation shown in Fig. 4.

Therefore, a different impedance controller will be formulated in the next section, which removes this drawback.

\section{COMBINED Design OF COMPLIANCE AND GRAVITY COMPENSATION}

In this section, the design idea for the gravity compensation from Section V-B is generalized by simultaneously taking account of the desired stiffness. This will result in an improved impedance control law that exactly implements the desired static stiffness relation.

\section{A. Controller Design}

Consider the case that a constant torque $\tau_{\text {ext }}$ acts on the robot (4)-(5). The equilibrium conditions for this case are

$$
\begin{aligned}
& \boldsymbol{K}\left(\boldsymbol{\theta}_{0}-\boldsymbol{q}_{0}\right)=\boldsymbol{g}\left(\boldsymbol{q}_{0}\right)-\boldsymbol{\tau}_{\mathrm{ext}} \\
& \boldsymbol{K}\left(\boldsymbol{\theta}_{0}-\boldsymbol{q}_{0}\right)=\boldsymbol{u}_{0}
\end{aligned}
$$

where $\boldsymbol{u}_{0}$ is the static value of $\boldsymbol{u}$. In the following, the desired stiffness relation

$$
\boldsymbol{K}_{\theta}\left(\boldsymbol{q}_{0}-\boldsymbol{q}_{d}\right)=\boldsymbol{\tau}_{\mathrm{ext}}
$$

shall be achieved statically. By combining (36) with (34), one gets the condition:

$$
\boldsymbol{K}\left(\boldsymbol{\theta}_{0}-\boldsymbol{q}_{0}\right)=\boldsymbol{g}\left(\boldsymbol{q}_{0}\right)-\boldsymbol{K}_{\theta}\left(\boldsymbol{q}_{0}-\boldsymbol{q}_{d}\right) .
$$

This condition can be seen as a relationship between the static motor side position $\boldsymbol{\theta}_{0}$ and the static link side position $\boldsymbol{q}_{0}$. In order to stress the similarity of the following derivation to the derivation of the gravity compensation term in Section V-B, the function $l(q)$ is defined as:

$$
\boldsymbol{l}(\boldsymbol{q}):=\boldsymbol{g}(\boldsymbol{q})-\boldsymbol{K}_{\theta}\left(\boldsymbol{q}-\boldsymbol{q}_{d}\right) .
$$

The following procedure is then completely analogous to the design of the gravity compensation term in Section V-B. The function $\boldsymbol{l}(\boldsymbol{q})$ now plays the same role as the gravity function $\boldsymbol{g}(\boldsymbol{q})$ previously. Notice that (37) can also be written as $\boldsymbol{K}\left(\boldsymbol{\theta}_{0}-\right.$ $\left.\boldsymbol{q}_{0}\right)=\boldsymbol{l}\left(\boldsymbol{q}_{0}\right)$ and by defining the function

$$
\boldsymbol{h}_{l}(\boldsymbol{q}):=\boldsymbol{q}+\boldsymbol{K}^{-1} \boldsymbol{l}(\boldsymbol{q})
$$

the static motor side position $\boldsymbol{\theta}_{0}$ can be expressed as $\boldsymbol{\theta}_{0}=$ $\boldsymbol{h}_{l}\left(\boldsymbol{q}_{0}\right)$. At this point, it is assumed that the inverse function of $\boldsymbol{h}_{l}(\boldsymbol{q})$ exists and it will be denoted by

$$
\overline{\boldsymbol{q}}_{l}(\boldsymbol{\theta}):=\boldsymbol{h}_{l}^{-1}(\boldsymbol{\theta}) .
$$

A sufficient condition for the existence of this inverse function as well as an iterative computation procedure will be given later in Proposition 3. By means of $\overline{\boldsymbol{q}}_{l}(\boldsymbol{\theta})$, a control law combining the gravity compensation with a statically exact stiffness design can be designed in the form:

$$
\begin{aligned}
\boldsymbol{u} & =\boldsymbol{l}\left(\overline{\boldsymbol{q}}_{l}(\boldsymbol{\theta})\right)-\boldsymbol{D}_{\theta} \dot{\boldsymbol{\theta}} \\
& =\boldsymbol{g}\left(\overline{\boldsymbol{q}}_{l}(\boldsymbol{\theta})\right)-\boldsymbol{K}_{\theta}\left(\overline{\boldsymbol{q}}_{l}(\boldsymbol{\theta})-\boldsymbol{q}_{d}\right)-\boldsymbol{D}_{\theta} \dot{\boldsymbol{\theta}} .
\end{aligned}
$$

The function $\boldsymbol{l}(\boldsymbol{q})$, as defined in (38), is the differential of the potential function

$$
V_{l}(\boldsymbol{q})=V_{g}(\boldsymbol{q})-\frac{1}{2}\left(\boldsymbol{q}-\boldsymbol{q}_{d}\right)^{T} \boldsymbol{K}_{\theta}\left(\boldsymbol{q}-\boldsymbol{q}_{d}\right)
$$

i.e., $\boldsymbol{l}(\boldsymbol{q})=\left(\partial V_{l}(\boldsymbol{q}) / \partial \boldsymbol{q}\right)^{T}$. Instead of the Assumptions 1 and 2 , the following assumption is needed now.

Assumption 3: The Hessian $\boldsymbol{H}_{l}(\boldsymbol{q})=\partial^{2} V_{l}(\boldsymbol{q}) / \partial \boldsymbol{q}^{2}$ of the potential function $V_{l}(\boldsymbol{q})$ satisfies the condition:

$$
\alpha_{l}:=\sup _{\forall \boldsymbol{q} \in \mathcal{Q}^{p}}\left\|\boldsymbol{H}_{l}(\boldsymbol{q})\right\|_{K}<\|\boldsymbol{K}\|_{K}=1 .
$$

Notice that this assumption implicitly contains an upper bound on the desired stiffness $\boldsymbol{K}_{\theta}$, similar to Assumption 2 for the previous controller. This is not surprising since, again, the controller basically implements a stiffness, which is in series interconnection to the joint stiffness $\boldsymbol{K}$. The stiffness $\boldsymbol{K}_{\theta}$, therefore, must be smaller than $\boldsymbol{K}$. Assumption 3, however, ensures the existence of the inverse function $\boldsymbol{h}_{l}^{-1}(\boldsymbol{\theta})$ as formulated in the following proposition, which is analogous to Proposition 1. 
Proposition 3: If Assumption 3 holds globally (i.e., for $\mathcal{Q}^{p}=$ $\left.\mathbb{R}^{n}\right)$, then the inverse function $\boldsymbol{h}_{l}^{-1}(\boldsymbol{\theta}):=\overline{\boldsymbol{q}}_{l}(\boldsymbol{\theta})$ of $\boldsymbol{h}_{l}(\boldsymbol{q})=\boldsymbol{q}+$ $\boldsymbol{K}^{-1} \boldsymbol{l}(\boldsymbol{q}): \mathbb{R}^{n} \rightarrow \mathbb{R}^{n}$ exists globally. Moreover, the iteration

$$
\hat{\boldsymbol{q}}_{l, n+1}=\boldsymbol{T}_{l}\left(\hat{\boldsymbol{q}}_{l, n}\right)
$$

with $\boldsymbol{T}_{l}(\boldsymbol{q}):=\boldsymbol{\theta}-\boldsymbol{K}^{-1} \boldsymbol{l}(\boldsymbol{q})$ converges for every fixed $\boldsymbol{\theta}$ and for every starting point $\hat{\boldsymbol{q}}_{l, 0}$ to $\overline{\boldsymbol{q}}_{l}(\boldsymbol{\theta})$.

Furthermore, by following the same derivation as in the Appendix [with $\boldsymbol{l}(\boldsymbol{q})$ instead of $\boldsymbol{g}(\boldsymbol{q})$ ], one can show that the controller term $\boldsymbol{l}\left(\overline{\boldsymbol{q}}_{l}(\boldsymbol{\theta})\right)$ can be written as the differential of the potential function

$$
\begin{aligned}
V_{\bar{l}}(\boldsymbol{\theta}) & =V_{l}\left(\overline{\boldsymbol{q}}_{l}(\boldsymbol{\theta})\right)+\frac{1}{2} \boldsymbol{l}\left(\overline{\boldsymbol{q}}_{l}(\boldsymbol{\theta})\right)^{T} \boldsymbol{K}^{-1} \boldsymbol{l}\left(\overline{\boldsymbol{q}}_{l}(\boldsymbol{\theta})\right) \\
\text { i.e., } \boldsymbol{l}\left(\overline{\boldsymbol{q}}_{l}(\boldsymbol{\theta})\right) & =\left(\partial V_{\bar{l}}(\boldsymbol{\theta}) / \partial \boldsymbol{\theta}\right)^{T} .
\end{aligned}
$$

\section{B. Stability Analysis}

The closed-loop system for the controller (41) together with (3) is given by:

$$
\begin{aligned}
\boldsymbol{M}(\boldsymbol{q}) \ddot{\boldsymbol{q}}+\boldsymbol{C}(\boldsymbol{q}, \dot{\boldsymbol{q}}) \dot{\boldsymbol{q}}+\boldsymbol{g}(\boldsymbol{q}) & =\boldsymbol{K}(\boldsymbol{\theta}-\boldsymbol{q})+\boldsymbol{\tau}_{\mathrm{ext}} \\
\boldsymbol{B}_{\theta} \ddot{\boldsymbol{\theta}}+\boldsymbol{K}(\boldsymbol{\theta}-\boldsymbol{q}) & =\boldsymbol{l}\left(\overline{\boldsymbol{q}}_{l}(\boldsymbol{\theta})\right)-\boldsymbol{D}_{\theta} \dot{\boldsymbol{\theta}} .
\end{aligned}
$$

Following the same line of argumentation as in the previous section, one can also prove the asymptotic stability for this system by using the Lyapunov function

$$
\begin{aligned}
V_{e}(\boldsymbol{q}, \dot{\boldsymbol{q}}, \boldsymbol{\theta}, \dot{\boldsymbol{\theta}})= & \frac{1}{2} \dot{\boldsymbol{q}}^{T} \boldsymbol{M}(\boldsymbol{q}) \dot{\boldsymbol{q}}+\frac{1}{2} \dot{\boldsymbol{\theta}}^{T} \boldsymbol{B}_{\theta} \dot{\boldsymbol{\theta}} \\
& +V_{g}(\boldsymbol{q})+V_{k}(\boldsymbol{q}, \boldsymbol{\theta})-V_{\bar{l}}(\boldsymbol{\theta})
\end{aligned}
$$

with $V_{k}(\boldsymbol{q}, \boldsymbol{\theta})$ and $V_{\bar{l}}(\boldsymbol{\theta})$ given in (33) and (45). This is summarized in the following proposition.

Proposition 4: Under the Assumption 3, the system (46)(47) is asymptotically stable for the case of free motion (i.e., for $\boldsymbol{\tau}_{\text {ext }}=\mathbf{0}$ ). Moreover, if Assumption 3 holds globally (i.e., for $\mathcal{Q}^{p}=\mathbb{R}^{n}$ ), then the system is even globally asymptotically stable. Considering interaction with the environment, i.e., for $\boldsymbol{\tau}_{\text {ext }} \neq \mathbf{0}$, the closed-loop system represents a passive mapping $\tau_{\mathrm{ext}} \rightarrow \dot{\boldsymbol{q}}$.

\section{Controller Discussion}

Also notice that the control law presented in this section does not exactly implement the desired impedance (7), cf. Remark 3. However, this yields a good approximation, which improves with higher inner loop torque feedback. In the experimental part in Section VIII, some comparisons with a simulation of the desired impedance are presented, which give an impression how well the desired impedance is approximated. But in contrast to the previous solution from Section $\mathrm{V}$, this controller now exactly fulfills the required steady-state condition. This can be seen by computing the steady state for a constant external torque $\tau_{\text {ext }}$, which leads to:

$$
\begin{aligned}
& \boldsymbol{K}\left(\boldsymbol{\theta}_{0}-\boldsymbol{q}_{0}\right)=\boldsymbol{g}\left(\boldsymbol{q}_{0}\right)-\boldsymbol{\tau}_{\mathrm{ext}} \\
& \boldsymbol{K}\left(\boldsymbol{\theta}_{0}-\boldsymbol{q}_{0}\right)=\boldsymbol{g}\left(\overline{\boldsymbol{q}}_{l}\left(\boldsymbol{\theta}_{0}\right)\right)-\boldsymbol{K}_{\theta}\left(\overline{\boldsymbol{q}}_{l}\left(\boldsymbol{\theta}_{0}\right)-\boldsymbol{q}_{d}\right) .
\end{aligned}
$$

Since $\overline{\boldsymbol{q}}_{l}(\boldsymbol{\theta})$ (by construction) satisfies (37), it follows that $\overline{\boldsymbol{q}}_{l}\left(\boldsymbol{\theta}_{0}\right)=\boldsymbol{q}_{0}$ must hold. This implies, as desired, $\boldsymbol{K}_{\theta}\left(\boldsymbol{q}_{0}-\right.$ $\left.\boldsymbol{q}_{d}\right)=\boldsymbol{\tau}_{\text {ext }}$.

At first glance, it might be somehow surprising that the controller is formulated in the coordinates $\overline{\boldsymbol{q}}_{l}(\boldsymbol{\theta})$ but does not require the Jacobian matrix $\partial \overline{\boldsymbol{q}}_{l}(\boldsymbol{\theta}) / \partial \boldsymbol{\theta}$ explicitly. Notice that the reason for this is that the function $\boldsymbol{l}\left(\overline{\boldsymbol{q}}_{l}(\boldsymbol{\theta})\right)$ is already the differential of the potential function $V_{\bar{l}}(\boldsymbol{\theta})$.

\section{GeneralizATIONS}

In the previous sections, two joint level impedance controllers were presented. Several extensions of these controllers are possible. Some of them are discussed in the following.

\section{A. Viscoelastic Joints}

Since the analysis of the controller was based on a physical interpretation of the torque feedback, it is also possible to include joint damping, i.e., gear damping, very easily. The considered model with joint damping is given by

$$
\begin{aligned}
\boldsymbol{M}(\boldsymbol{q}) \ddot{\boldsymbol{q}}+\boldsymbol{C}(\boldsymbol{q}, \dot{\boldsymbol{q}}) \dot{\boldsymbol{q}}+\boldsymbol{g}(\boldsymbol{q})= & \boldsymbol{K}(\boldsymbol{\theta}-\boldsymbol{q}) \\
& +\boldsymbol{D}(\dot{\boldsymbol{\theta}}-\dot{\boldsymbol{q}})+\boldsymbol{\tau}_{\mathrm{ext}} \\
\boldsymbol{B} \ddot{\boldsymbol{\theta}}+\boldsymbol{K}(\boldsymbol{\theta}-\boldsymbol{q})+\boldsymbol{D}(\dot{\boldsymbol{\theta}}-\dot{\boldsymbol{q}})= & \boldsymbol{\tau}_{m}
\end{aligned}
$$

where the matrix $\boldsymbol{D} \in \mathbb{R}^{n \times n}$ is a diagonal and positive definite damping matrix. For this model, the same type of controller as in the last section can be used, when the control law (3) is replaced by

$$
\boldsymbol{\tau}_{m}=\boldsymbol{B} \boldsymbol{B}_{\theta}^{-1} \boldsymbol{u}+\left(\boldsymbol{I}-\boldsymbol{B} \boldsymbol{B}_{\theta}^{-1}\right)\left(\boldsymbol{\tau}+\boldsymbol{D} \boldsymbol{K}^{-1} \dot{\boldsymbol{\tau}}\right)
$$

with $\boldsymbol{\tau}=\boldsymbol{K}(\boldsymbol{\theta}-\boldsymbol{q})$. This leads to the closed-loop system

$$
\begin{gathered}
\boldsymbol{M}(\boldsymbol{q}) \ddot{\boldsymbol{q}}+\boldsymbol{C}(\boldsymbol{q}, \dot{\boldsymbol{q}}) \dot{\boldsymbol{q}}+\boldsymbol{g}(\boldsymbol{q})=\boldsymbol{\tau}_{\mathrm{ext}}+\boldsymbol{K}(\boldsymbol{\theta}-\boldsymbol{q})+\boldsymbol{D}(\dot{\boldsymbol{\theta}}-\dot{\boldsymbol{q}}) \\
\boldsymbol{B}_{\theta} \ddot{\boldsymbol{\theta}}+\boldsymbol{K}(\boldsymbol{\theta}-\boldsymbol{q})+\boldsymbol{D}(\dot{\boldsymbol{\theta}}-\dot{\boldsymbol{q}})=\boldsymbol{u}
\end{gathered}
$$

for which the intermediate control input $\boldsymbol{u}$ can be chosen in the same way as in the previous sections. All the passivity and stability statements given in this paper also hold for a model with viscoelastic joints.

\section{B. Cartesian Impedance Control}

In many applications, the desired impedance behavior is defined with respect to the end-effector motion rather than in joint coordinates. In this section, it is shown that the controller from Section VI can easily be generalized to the implementation of a desired Cartesian impedance controller. In the Cartesian case, however, the singularities of the Jacobian matrix clearly pose a limitation on the achievable region of attraction. Also, for a Cartesian controller applied to a redundant robot, stability can only be achieved if the desired Cartesian behavior is augmented by some nullspace behavior. Despite these general differences between joint level control and Cartesian control, the generalization of the impedance controller to the Cartesian case can follow the same line of argumentation as in Section VI.

In the following, it is assumed that the forward kinematics mapping from the joint space coordinates $\boldsymbol{q}$ to the 
Cartesian coordinates $\boldsymbol{x}=\boldsymbol{f}(\boldsymbol{q}) \in \mathbb{R}^{6}$ as well as the Jacobian matrix $\boldsymbol{J}(\boldsymbol{q})=\partial \boldsymbol{f}(\boldsymbol{q}) / \partial \boldsymbol{q} \in \mathbb{R}^{6 \times n}$ are known. The desired impedance behavior is specified in terms of a Cartesian virtual equilibrium position $\boldsymbol{x}_{d}$, a symmetric and positive definite stiffness matrix $\boldsymbol{K}_{x} \in \mathbb{R}^{6 \times 6}$, and a positive definite damping matrix $\boldsymbol{D}_{x} \in \mathbb{R}^{6 \times 6}$. Based on this, one can formulate a desired Cartesian stiffness potential in the form:

$$
V_{x}=\frac{1}{2}\left(\boldsymbol{x}-\boldsymbol{x}_{d}\right)^{T} \boldsymbol{K}_{x}\left(\boldsymbol{x}-\boldsymbol{x}_{d}\right) .
$$

Consider the case that a constant generalized external force $\boldsymbol{F}_{\text {ext }}$ acts on the robot. In steady state at a position $\boldsymbol{q}_{0}$, the generalized external force $\boldsymbol{F}_{\text {ext }}$ is related to the external torques $\boldsymbol{\tau}_{\text {ext }}$ via $\boldsymbol{\tau}_{\text {ext }}=\boldsymbol{J}\left(\boldsymbol{q}_{0}\right)^{T} \boldsymbol{F}_{\text {ext }}$. The desired static equilibrium condition for this case is

$$
\boldsymbol{F}_{\text {ext }}=\boldsymbol{K}_{x}\left(\boldsymbol{x}_{0}-\boldsymbol{x}_{d}\right)=\left(\frac{\partial V_{x}(\boldsymbol{x})}{\partial \boldsymbol{x}}\right)_{\boldsymbol{x}=\boldsymbol{x}_{0}}^{T}
$$

which can be equivalently expressed in joint coordinates as

$$
\boldsymbol{\tau}_{\text {ext }}=\left(\frac{\mathrm{d} V_{x}(\boldsymbol{f}(\boldsymbol{q}))}{\mathrm{d} \boldsymbol{q}}\right)_{\boldsymbol{q}=\boldsymbol{q}_{0}}^{T}
$$

as long as the Jacobian matrix remains nonsingular. By combining this desired steady-state condition with (34), one now gets [instead of (37)] the equation:

$$
\boldsymbol{K}\left(\boldsymbol{\theta}_{0}-\boldsymbol{q}_{0}\right)=\boldsymbol{g}\left(\boldsymbol{q}_{0}\right)-\left(\frac{\mathrm{d} V_{x}(\boldsymbol{f}(\boldsymbol{q}))}{\mathrm{d} \boldsymbol{q}}\right)_{\boldsymbol{q}=\boldsymbol{q}_{0}}^{T} .
$$

The terms on the right-hand side of this equation motivate the definition of the function

$$
\boldsymbol{c}(\boldsymbol{q}):=\boldsymbol{g}(\boldsymbol{q})-\left(\frac{\mathrm{d} V_{x}(\boldsymbol{f}(\boldsymbol{q}))}{\mathrm{d} \boldsymbol{q}}\right)^{T} .
$$

which replaces $\boldsymbol{l}(\boldsymbol{q})$ from Section VI. For completing the controller design, one can then repeat the procedure from the joint level case using $\boldsymbol{c}(\boldsymbol{q})$ instead of $\boldsymbol{l}(\boldsymbol{q})$ as well as $V_{c}(\boldsymbol{q})=$ $V_{g}(\boldsymbol{q})-V_{x}(\boldsymbol{q})$ instead of $V_{l}(\boldsymbol{q})$. Consequently, the controller can be formulated as

$$
\begin{aligned}
\boldsymbol{u} & =\boldsymbol{c}\left(\overline{\boldsymbol{q}}_{c}(\boldsymbol{\theta})\right)+\boldsymbol{D}_{c}(\boldsymbol{\theta}) \dot{\boldsymbol{\theta}} \\
& =\boldsymbol{g}\left(\overline{\boldsymbol{q}}_{c}(\boldsymbol{\theta})\right)-\left(\frac{\mathrm{d} V_{c}(\boldsymbol{f}(\boldsymbol{q}))}{\mathrm{d} \boldsymbol{q}}\right)_{\boldsymbol{q}=\overline{\boldsymbol{q}}_{c}(\boldsymbol{\theta})}^{T}+\boldsymbol{D}_{c}(\boldsymbol{\theta}) \dot{\boldsymbol{\theta}}
\end{aligned}
$$

where $\overline{\boldsymbol{q}}_{c}(\boldsymbol{\theta})$ corresponds to the solution of the equation $\boldsymbol{K}(\boldsymbol{\theta}$ $\boldsymbol{q})=\boldsymbol{c}(\boldsymbol{q})$ for $\boldsymbol{q}$ and $\boldsymbol{D}_{c}(\boldsymbol{\theta})$ is a joint level damping matrix chosen as

$$
\boldsymbol{D}_{c}(\boldsymbol{\theta})=\boldsymbol{J}\left(\overline{\boldsymbol{q}}_{c}(\boldsymbol{\theta})\right)^{T} \boldsymbol{D}_{x} \boldsymbol{J}\left(\overline{\boldsymbol{q}}_{c}(\boldsymbol{\theta})\right)
$$

which is positive definite as long as the Jacobian matrix is nonsingular. For ensuring the existence and uniqueness of $\overline{\boldsymbol{q}}_{c}(\boldsymbol{\theta})$, the following assumption is needed representing an upper bound of the achievable Cartesian stiffness.

Assumption 4: The Hessian $\boldsymbol{H}_{c}(\boldsymbol{q})=\partial^{2} V_{c}(\boldsymbol{q}) / \partial \boldsymbol{q}^{2}$ of the potential function $V_{c}(\boldsymbol{q})$ satisfies the condition:

$$
\alpha_{c}:=\sup _{\forall \boldsymbol{q} \in \mathcal{Q}^{p}}\left\|\boldsymbol{H}_{c}(\boldsymbol{q})\right\|_{K}<\|\boldsymbol{K}\|_{K}=1 .
$$

This assumption implicitly represents an upper bound for the Cartesian stiffness $\boldsymbol{K}_{x}$ with respect to the joint stiffness $\boldsymbol{K}$ and is analogous to Assumption 3 from the joint level controller. It ensures the existence of $\overline{\boldsymbol{q}}_{c}(\boldsymbol{\theta})$ according to the following proposition.

Proposition 5: If Assumption 4 holds globally (i.e., for $\mathcal{Q}^{p}=$ $\left.\mathbb{R}^{n}\right)$, then the function $\overline{\boldsymbol{q}}_{c}(\boldsymbol{\theta})$, i.e., the solution of $\boldsymbol{K}(\boldsymbol{\theta}-\boldsymbol{q})=$ $\boldsymbol{c}(\boldsymbol{q})$ for $\boldsymbol{q}$, exists globally. Moreover, the iteration

$$
\hat{\boldsymbol{q}}_{c, n+1}=\boldsymbol{T}_{c}\left(\hat{\boldsymbol{q}}_{c, n}\right)
$$

with $\boldsymbol{T}_{c}(\boldsymbol{q}):=\boldsymbol{\theta}-\boldsymbol{K}^{-1} \boldsymbol{c}(\boldsymbol{q})$ converges for every fixed $\boldsymbol{\theta}$ and for every starting point $\hat{\boldsymbol{q}}_{c, 0}$ to $\overline{\boldsymbol{q}}_{c}(\boldsymbol{\theta})$.

The above description presents the implementation of the controller so far. Notice that for the implementation, neither singularities of the Jacobian nor the redundant case are problematic since no inversion of the Jacobian is needed for the controller computation. The potential function for the controller is given by

$$
V_{\bar{c}}(\boldsymbol{\theta})=V_{c}\left(\overline{\boldsymbol{q}}_{c}(\boldsymbol{\theta})\right)+\frac{1}{2} \boldsymbol{c}\left(\overline{\boldsymbol{q}}_{c}(\boldsymbol{\theta})\right)^{T} \boldsymbol{K}^{-1} \boldsymbol{c}\left(\overline{\boldsymbol{q}}_{c}(\boldsymbol{\theta})\right)
$$

for which $\boldsymbol{c}\left(\overline{\boldsymbol{q}}_{c}(\boldsymbol{\theta})\right)=\left(\partial V_{\bar{c}}(\boldsymbol{\theta}) / \partial \boldsymbol{\theta}\right)^{T}$ holds. The control law again ensures passivity of the closed-loop system. This can be seen by using the positive semidefinite ${ }^{9}$ function

$$
\begin{aligned}
V_{f}(\boldsymbol{q}, \dot{\boldsymbol{q}}, \boldsymbol{\theta}, \dot{\boldsymbol{\theta}})= & \frac{1}{2} \dot{\boldsymbol{q}}^{T} \boldsymbol{M}(\boldsymbol{q}) \dot{\boldsymbol{q}}+\frac{1}{2} \dot{\boldsymbol{\theta}}^{T} \boldsymbol{B}_{\boldsymbol{\theta}} \dot{\boldsymbol{\theta}} \\
& +\boldsymbol{V}_{g}(\boldsymbol{q})+V_{k}(\boldsymbol{q}, \boldsymbol{\theta})-V_{\bar{c}}(\boldsymbol{\theta})
\end{aligned}
$$

as a storage function.

For proving stability, however, one must distinguish between the redundant and the nonredundant case. While (59) becomes positive definite for a nonredundant robot and can be used for proving (local ${ }^{10}$ ) asymptotic stability, an additional nullspace control is needed in the redundant case.

Regarding singularities of the orientation representation in the Cartesian coordinates $f(q)$, it should be mentioned that the potential function (49) could also be replaced by the potential of one of the singularity-free spatial springs proposed by, e.g., Fasse or Natale (see, e.g., [28], [29]).

\section{EXPERIMENTS}

In this section, some experiments are reported for evaluating the proposed controllers. The first two experiments were conducted with the 7 DOF DLR-Lightweight-Robot-II, while the second two were performed with the newer DLR-LightweightRobot-III. These robots are equipped with joint torque sensors additionally to the motor position sensors, and thus, are ideally suited for the implementation of the proposed controllers. For the experiments, the Cartesian control law from Section VII-B was chosen because it is the most complex controller from the paper and the interaction with the human user is then more intuitive. For the evaluation, a force-torque sensor was additionally mounted on the tip of the robots.

\footnotetext{
${ }^{9}$ The function is positive definite only in the nonredundant case.

${ }^{10}$ The global case is obstructed by the singularities of the Jacobian.
} 

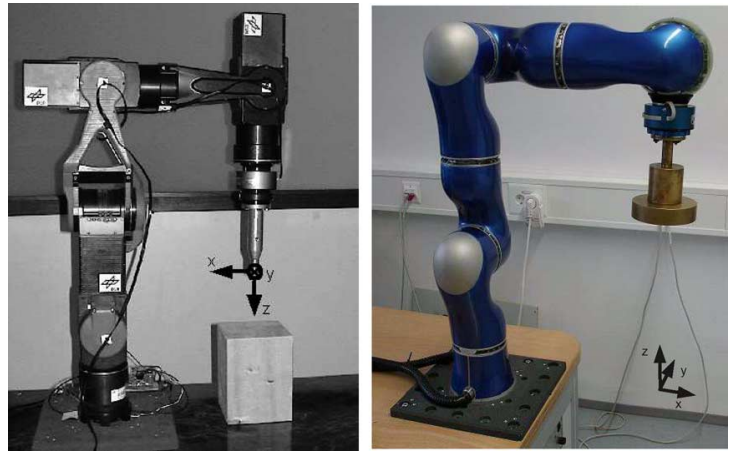

Fig. 6. Initial configuration of the DLR lightweight robots (LWR-II left, LWRIII right) for the experiments.

TABLE I

STIFFNESS AND DAMPING VALUES FOR THE FIRST EXPERIMENT

\begin{tabular}{|l|c|c|c|c|c|c|}
\hline Coord. & $e_{x}$ & $e_{y}$ & $e_{z}$ & $\phi_{x}$ & $\phi_{y}$ & $\phi_{z}$ \\
\hline Stiffness & 700 & 4000 & 4000 & 200 & 200 & 200 \\
& $\frac{N}{m}$ & $\frac{N}{m}$ & $\frac{N}{m}$ & $\frac{N m}{r a d}$ & $\frac{N m}{r a d}$ & $\frac{N m}{r a d}$ \\
\hline Damping & 70 & 400 & 400 & 5 & 5 & 5 \\
& $\frac{N s}{m}$ & $\frac{N s}{m}$ & $\frac{N s}{m}$ & $\frac{N m s}{r a d}$ & $\frac{N m s}{r a d}$ & $\frac{N m s}{r a d}$ \\
\hline
\end{tabular}

Fig. 6 shows the initial configuration of the robots for the experiments. In the first experiment, the achieved compliance is evaluated. The Cartesian impedance from Section VII-B was implemented with diagonal stiffness and damping matrices with the values given in Table I. The three translational coordinates are denoted by $e_{x}, e_{y}$, and $e_{z}$. For the orientation representation, RPY Euler angles were used. The orientational coordinates are denoted by $\phi_{x}, \phi_{y}$, and $\phi_{z}$.

In the experiment, a human user exerts (generalized) forces on the robot end-effector by pulling and pushing, mainly in the horizontal ( $x$ - and $y$-coordinates) directions. The interaction forces are measured by a 6 DOF force-torque sensor ${ }^{11}$ mounted on the end-effector. Notice that this sensor was not used in the implementation of the impedance controller but is used only for evaluation purposes. The applied forces in $x$ - and $y$-direction over time are shown in Fig. 7. In order to evaluate the resulting stiffness and damping, the force and displacement in $x$ - and $y$-direction are shown in Figs. 8 and 9, respectively. The corresponding static characteristic line according to the relevant stiffness value from Table I is shown by the dashed line. Notice that the hysteresis-like deviation from the static value is caused by the Cartesian damping. Additionally, the dotted line shows the result of a simple simulation of the desired Cartesian impedance. In this simulation, the measured contact force is used as an input and the Cartesian motion is the output. This simulation contains some further simplifications. ${ }^{12}$ Notice that the simulation shows only the desired compliance and no joint elasticity is included. One can see that the experimental results fit quite well the simulation of the desired compliance for low (see Fig. 8) and high (see Fig. 9) Cartesian stiffness values.

\footnotetext{
${ }^{11}$ A JR3 sensor was used.

${ }^{12}$ The inertia matrix was considered constant and, accordingly, no centrifugal and Coriolis terms were included.
}

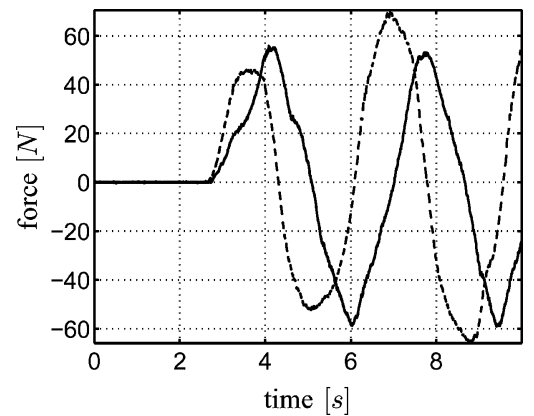

Fig. 7. Applied forces in $x$-direction (solid line) and $y$-direction (dashed line) in the first experiment.

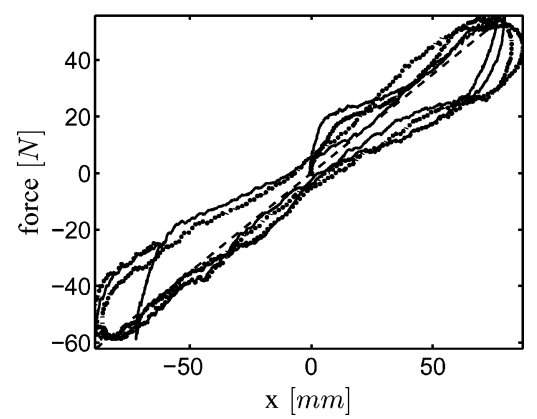

Fig. 8. Applied force versus end-effector deviation in $x$-direction (solid line). The dashed line corresponds to the desired stiffness. The dotted line shows a (simplified) simulation result.

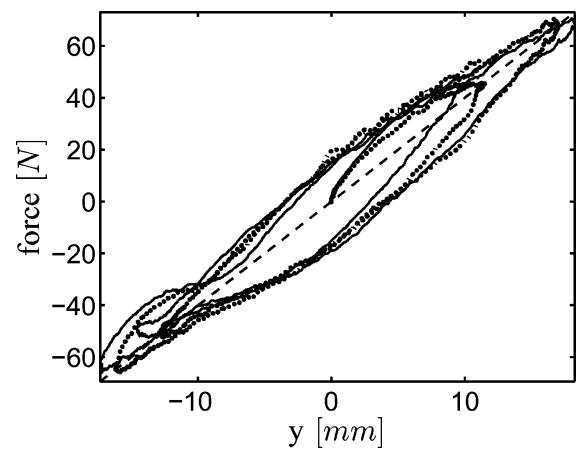

Fig. 9. Applied force versus end-effector deviation in $y$-direction (solid line). The dashed line corresponds to the desired stiffness. The dotted line shows a (simplified) simulation result.

In a second experiment, an impact with a wooden surface was performed using the controller from Section VII-B with the parameters from Table II. This experiment shows the robustness of the controller in contact with a passive environment. The initial configuration is shown in Fig. 6. Fig. 10 displays the desired and the measured end-effector motion in the vertical $z$-direction during the impact. Additionally, Fig. 11 depicts the impact force. The measured static end-effector deviation and contact force give a stiffness value of $\sim 3882 \mathrm{~N} / \mathrm{m}$, which corresponds very well to the desired value of $4000 \mathrm{~N} / \mathrm{m}$. The peak of the contact force results mainly from the velocity at the impact. One can see that the closed-loop system also keeps stable in contact with this environment having quite a high stiffness, and that, high impact velocities can also be handled. 
TABLE II

STIFFNESS AND DAMPING VALUES FOR THE IMPACT EXPERIMENT

\begin{tabular}{|l|c|c|c|c|c|c|}
\hline Coord. & $e_{x}$ & $e_{y}$ & $e_{z}$ & $\phi_{x}$ & $\phi_{y}$ & $\phi_{z}$ \\
\hline Stiffness & 4000 & 4000 & 4000 & 200 & 200 & 200 \\
& $\frac{N}{m}$ & $\frac{N}{m}$ & $\frac{N}{m}$ & $\frac{N m}{r a d}$ & $\frac{N m}{r a d}$ & $\frac{N m}{r a d}$ \\
\hline Damping & 400 & 400 & 400 & 5 & 5 & 5 \\
& $\frac{N s}{m}$ & $\frac{N s}{m}$ & $\frac{N s}{m}$ & $\frac{N m s}{r a d}$ & $\frac{N m s}{r a d}$ & $\frac{N m s}{r a d}$ \\
\hline
\end{tabular}

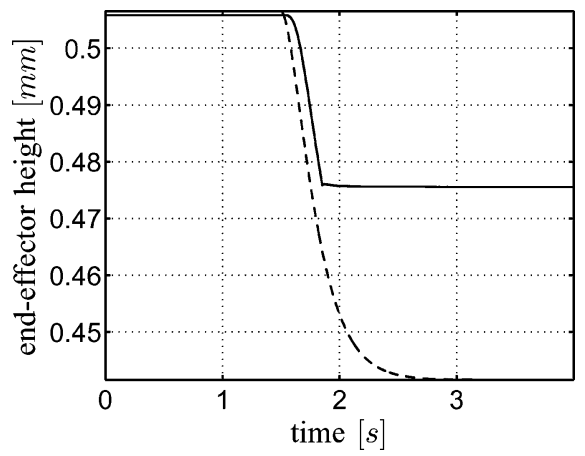

Fig. 10. End-effector height in the impact experiment. The dashed line shows the end-effector height of the virtual equilibrium position and the solid line the measured end-effector position.

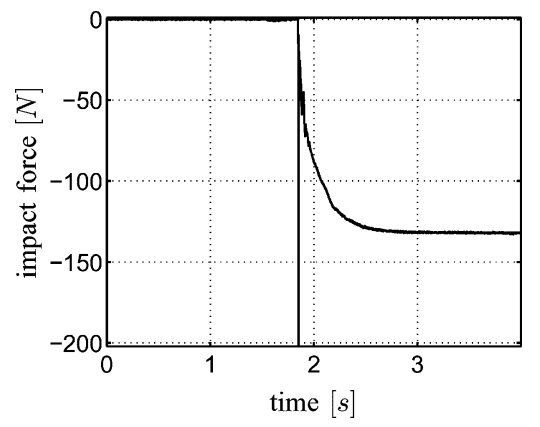

Fig. 11. Measured force in the impact experiment.

Two additional experiments with the DLR-LightweightRobot-III were performed in order to analyze the step response of the Cartesian controller as well as the effects of uncertainties in the end-effector load. The stiffness values for these experiments were chosen smaller than in the first two experiments and are given in Table III. In this experiment, a heavy load of about $4.5 \mathrm{~kg}$ was attached to the end-effector.

In this evaluation, only the Cartesian position will be analyzed. In Fig. 12, the step response for a step of $30 \mathrm{~mm}$ in vertical $z$-direction is shown with and without the load attached. In both cases, the exact load was known for the controller computation. Since the controller does not shape the effective inertia but implements stiffness and damping, the step response changes accordingly. The remaining end-effector deviation is in the range of the known friction effects for this robot. Next, the effects of uncertainties in the load shall be analyzed. Notice that the controller does only contain the gravity model, while it does not require the computation of the inertia matrix or the centrifugal and Coriolis terms. Again, the load of $\sim 4.5 \mathrm{~kg}$ was attached to the end-effector. At the beginning of the experiment, the load is included in the computation of the controller. Then, at time step $t=0.26 \mathrm{~s}$, the load in the controller computation is
TABLE III

STIFFNESS AND DAMPING VALUES FOR THE STEP RESPONSE EXPERIMENT

\begin{tabular}{|l|c|c|c|c|c|c|}
\hline Coord. & $e_{x}$ & $e_{y}$ & $e_{z}$ & $\phi_{x}$ & $\phi_{y}$ & $\phi_{z}$ \\
\hline Stiffness & 2000 & 2000 & 2000 & 100 & 100 & 100 \\
& $\frac{N}{m}$ & $\frac{N}{m}$ & $\frac{N}{m}$ & $\frac{N m}{r a d}$ & $\frac{N m}{r a d}$ & $\frac{N m}{r a d}$ \\
\hline Damping & 110 & 110 & 110 & 14 & 14 & 14 \\
& $\frac{N s}{m}$ & $\frac{N s}{m}$ & $\frac{N s}{m}$ & $\frac{N m s}{r a d}$ & $\frac{N m s}{r a d}$ & $\frac{N m s}{r a d}$ \\
\hline
\end{tabular}

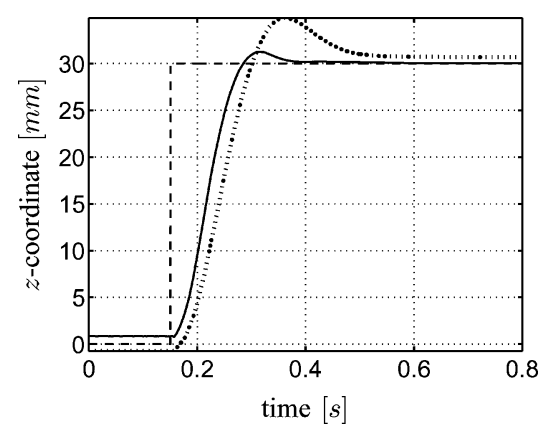

Fig. 12. Step response for the Cartesian impedance controller. The dashed line shows the commanded step. The step response with and without load are shown by the dotted and solid line, respectively.

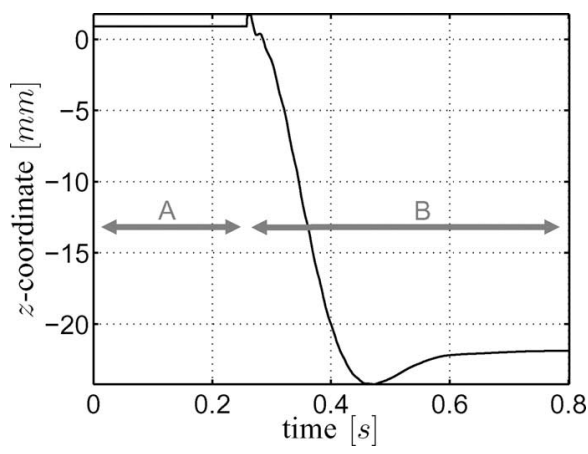

Fig. 13. Effectof uncertainties in the gravity model. In time period A, the load $(\sim 4.5 \mathrm{~kg})$ of the end-effector is known and considered in the controller, while in time period $\mathrm{B}$, this information is not included.

set to zero simulating a huge model uncertainty for the gravity compensation. Fig. 13 shows the Cartesian position deviations for the case of the known (time period A) and unknown (time period B) load. One can see that the deviation in time period B corresponds very well to the commanded stiffness of $2000 \mathrm{~N} / \mathrm{m}$ with an external force resulting from the unknown load. While uncertainties in the load thus clearly affect the position accuracy according to the desired stiffness behavior, the stability of the system is not affected by this large model error. This goes in accordance with the theoretically proven (passivity-based) robustness properties.

\section{SUMmary}

In this paper, we propose two impedance controllers for flexible joint robots. In both controllers, an inner torque feedback loop is used in combination with an outer impedance control loop. For the torque feedback, a physical interpretation is given, such that the complete controllers could be analyzed based on passivity theory.

The first controller combines a motor-position-based gravity compensation term with a stiffness and damping term. In 
the second controller, these parts are instead merged such that at steady state, the desired equilibrium condition could be satisfied exactly. It is shown that both controllers can easily be adapted to the case of viscoelastic joints. Furthermore, the generalization to Cartesian impedance control has been outlined. Finally, the efficiency of the proposed control approach was verified in several experiments with the DLR lightweight robots.

\section{APPENDIX}

In this appendix, the potential function $V_{\bar{g}}(\boldsymbol{\theta})$ for the gravity compensation term $\overline{\boldsymbol{g}}(\boldsymbol{\theta})$ is derived such that $\overline{\boldsymbol{g}}(\boldsymbol{\theta})=$ $\left(\partial V_{\bar{g}}(\boldsymbol{\theta}) / \partial \boldsymbol{\theta}\right)^{T}$ holds. Remember that for the construction of $\overline{\boldsymbol{g}}(\boldsymbol{\theta})=\boldsymbol{g}(\overline{\boldsymbol{q}}(\boldsymbol{\theta}))$ in Section V-B, the function $\overline{\boldsymbol{q}}(\boldsymbol{\theta})=\boldsymbol{h}_{g}^{-1}(\boldsymbol{\theta})$, i.e., the inverse of the function $\boldsymbol{h}_{g}(\boldsymbol{q})=\boldsymbol{q}+\boldsymbol{K}^{-1} \boldsymbol{g}(\boldsymbol{q})$, was used. Existence and uniqueness of $\boldsymbol{h}_{g}^{-1}(\boldsymbol{\theta})$ were established in Proposition 1 by the use of Assumption 1 .

In the following, the Jacobian matrix $\partial \overline{\boldsymbol{q}}(\boldsymbol{\theta}) / \partial \boldsymbol{\theta}$ will be needed. Consider first the Jacobian matrix of the function $\boldsymbol{h}_{g}(\boldsymbol{q})$

$$
\frac{\partial \boldsymbol{h}_{g}(\boldsymbol{q})}{\boldsymbol{q}}=\left(\boldsymbol{I}+\boldsymbol{K}^{-1} \frac{\partial \boldsymbol{g}(\boldsymbol{q})}{\partial \boldsymbol{q}}\right) .
$$

Due to $\boldsymbol{h}_{g}(\overline{\boldsymbol{q}}(\boldsymbol{\theta}))=\boldsymbol{\theta}$, one has

$$
\frac{\partial \boldsymbol{h}_{g}(\overline{\boldsymbol{q}}(\boldsymbol{\theta}))}{\partial \boldsymbol{\theta}}=\frac{\partial \boldsymbol{h}_{g}(\overline{\boldsymbol{q}})}{\partial \overline{\boldsymbol{q}}} \frac{\partial \overline{\boldsymbol{q}}(\boldsymbol{\theta})}{\partial \boldsymbol{\theta}}=\boldsymbol{I},
$$

and therefore, the Jacobian matrix $\partial \overline{\boldsymbol{q}}(\boldsymbol{\theta}) / \partial \boldsymbol{\theta}$ is given by:

$$
\frac{\partial \overline{\boldsymbol{q}}(\boldsymbol{\theta})}{\partial \boldsymbol{\theta}}=\left(\boldsymbol{I}+\boldsymbol{K}^{-1} \frac{\partial \boldsymbol{g}(\overline{\boldsymbol{q}})}{\partial \overline{\boldsymbol{q}}}\right)_{\overline{\boldsymbol{q}}=\overline{\boldsymbol{q}}(\boldsymbol{\theta})}^{-1} .
$$

The potential function $V_{\bar{g}}(\boldsymbol{\theta})$ clearly can be written in the form

$$
V_{\bar{g}}(\boldsymbol{\theta})=V_{\bar{g}}\left(\boldsymbol{h}_{g}(\overline{\boldsymbol{q}}(\boldsymbol{\theta}))\right)=: V_{\bar{g} h}(\overline{\boldsymbol{q}}(\boldsymbol{\theta})) .
$$

For the differential $\partial V_{\bar{g}}(\boldsymbol{\theta}) / \partial \boldsymbol{\theta}$, one obtains:

$$
\frac{\partial V_{\bar{g}}(\boldsymbol{\theta})}{\partial \boldsymbol{\theta}}=\left(\frac{\partial V_{\bar{g} h}(\overline{\boldsymbol{q}})}{\partial \overline{\boldsymbol{q}}}\right)_{\overline{\boldsymbol{q}}=\overline{\boldsymbol{q}}(\boldsymbol{\theta})} \frac{\partial \overline{\boldsymbol{q}}(\boldsymbol{\theta})}{\partial \boldsymbol{\theta}} .
$$

By substituting $\quad V_{\partial \bar{g}}(\boldsymbol{\theta}) / \partial \boldsymbol{\theta}=\overline{\boldsymbol{g}}(\boldsymbol{q})=\boldsymbol{g}(\overline{\boldsymbol{q}}(\boldsymbol{\theta}))^{T}$ and $\partial \overline{\boldsymbol{q}}(\boldsymbol{\theta}) / \partial \boldsymbol{\theta}$ from (61), one gets:

$$
\begin{aligned}
\frac{\partial V_{\bar{g} h}(\overline{\boldsymbol{q}})}{\partial \overline{\boldsymbol{q}}} & =\boldsymbol{g}(\overline{\boldsymbol{q}})^{T}\left(\boldsymbol{I}+\boldsymbol{K}^{-1} \frac{\partial \boldsymbol{g}(\overline{\boldsymbol{q}})}{\partial \overline{\boldsymbol{q}}}\right) \\
& =\boldsymbol{g}(\overline{\boldsymbol{q}})^{T}+\boldsymbol{g}(\overline{\boldsymbol{q}})^{T} \boldsymbol{K}^{-1} \frac{\partial \boldsymbol{g}(\overline{\boldsymbol{q}})}{\partial \overline{\boldsymbol{q}}} .
\end{aligned}
$$

This differential can be integrated to $V_{\bar{g} h}(\overline{\boldsymbol{q}})=V_{g}(\overline{\boldsymbol{q}})+$ $\frac{1}{2} \boldsymbol{g}(\overline{\boldsymbol{q}})^{T} \boldsymbol{K}^{-1} \boldsymbol{g}(\overline{\boldsymbol{q}})+\boldsymbol{c}$ with an arbitrary constant $\boldsymbol{c} \in \mathbb{R}^{n}$ and the gravity potential $V_{g}(\boldsymbol{q})$ from Section IV. Setting $\boldsymbol{c}=\mathbf{0}$ leads to the gravity compensation potential:

$$
\begin{aligned}
V_{\bar{g}}(\boldsymbol{\theta}) & =V_{\bar{g} h}(\overline{\boldsymbol{q}}(\boldsymbol{\theta})) \\
& =V_{g}(\overline{\boldsymbol{q}}(\boldsymbol{\theta}))+\frac{1}{2} \boldsymbol{g}(\overline{\boldsymbol{q}}(\boldsymbol{\theta}))^{T} \boldsymbol{K}^{-1} \boldsymbol{g}(\overline{\boldsymbol{q}}(\boldsymbol{\theta})) .
\end{aligned}
$$

Also notice that for all stationary points, the potential energy of the manipulator $V_{\text {pot }}(\boldsymbol{q}, \boldsymbol{\theta})=V_{k}(\boldsymbol{q}, \boldsymbol{\theta})+V_{g}(\boldsymbol{q})$, with $V_{k}(\boldsymbol{q}, \boldsymbol{\theta})=\frac{1}{2}(\boldsymbol{\theta}-\boldsymbol{q})^{T} \boldsymbol{K}(\boldsymbol{\theta}-\boldsymbol{q})$ as the potential of the joint stiffness, is identical to the gravity compensation potential, i.e.,

$$
V_{\text {pot }}(\boldsymbol{q}, \boldsymbol{\theta})=V_{\bar{g}}(\boldsymbol{\theta}) \quad \forall(\boldsymbol{q}, \boldsymbol{\theta}) \in \Omega .
$$

>From this, it follows that $V_{\bar{g}}(\boldsymbol{\theta})$ can also be written as:

$$
V_{\bar{g}}(\boldsymbol{\theta})=V_{\mathrm{pot}}(\overline{\boldsymbol{q}}(\boldsymbol{\theta}), \boldsymbol{\theta})=V_{g}(\overline{\boldsymbol{q}}(\boldsymbol{\theta}))+V_{k}(\overline{\boldsymbol{q}}(\boldsymbol{\theta}), \boldsymbol{\theta}) .
$$

\section{REFERENCES}

[1] N. Hogan, "Impedance control: An approach to manipulation, part ITheory," ASME J. Dyn. Syst., Meas., Control, vol. 107, pp. 1-7, 1985.

[2] M. Spong, "Modeling and control of elastic joint robots," Trans. ASME: J. Dyn. Syst., Meas., Control, vol. 109, pp. 310-319, 1987.

[3] A. De Luca and P. Lucibello, "A general algorithm for dynamic feedback linearization of robots with elastic joints," in Proc. IEEE Int. Conf. Robot. Autom., 1998, pp. 504-510.

[4] M. Spong, K. Khorasani, and P. Kokotovic, "An integral manifold approach to feedback control of flexible joint robots," IEEE J. Robot. Autom., vol. 3, no. 4, pp. 291-300, Aug. 1987.

[5] A. Albu-Schäffer, Ch. Ott, and G. Hirzinger, "A unified passivity-based control framework for position, torque and impedance control of flexible joint robots," Int. J. Robot. Res., vol. 26, no. 1, pp. 23-39, Jan. 2007.

[6] S. Nicosia and P. Tomei, "Design of global tracking controllers for flexiblejoint robots," J. Robotic Syst., vol. 10, no. 6, pp. 835-846, 1993.

[7] T. Lin and A. Goldenberg, "Robust adaptive control of flexible joint robots with joint torque feedback," in Proc. IEEE Int. Conf. Robot. Autom., 1995, pp. 1229-1234.

[8] B. Brogliato, R. Ortega, and R. Lozano, "Global tracking controllers for flexible-joint manipulators: A comparative study," Automatica, vol. 31, no. 7, pp. 941-956, 1995.

[9] R. Ortega, R. Kelly, and A. Loria, "A class of output feedback globally stabilizing controllers for flexible joints robots," IEEE Trans. Robot. Automat., vol. 11, no. 5, pp. 766-770, Oct. 1995.

[10] P. Tomei, "A simple pd controller for robots with elastic joints," IEEE Trans. Automat. Control, vol. 35, no. 10, pp. 1208-1213, Oct. 1991.

[11] P. Goldsmith, B. Francis, and A. Goldenberg, "Stability of hybrid position/force control applied to manipulators with flexible joints," Int. J. Robot. Autom., vol. 14, no. 4, pp. 146-160, 1999.

[12] M. Spong, "Adaptive control of flexible joint manipulators," Syst. Control Lett., vol. 13, no. 1, pp. 15-21, 1989.

[13] A. Albu-Schäffer, Ch. Ott, U. Frese, and G. Hirzinger, "Cartesian impedance control of redundant robots: Recent results with the DLR-lightweight-arms," in Proc. IEEE Int. Conf. Robot. Autom., 2003, pp. 3704 3709 .

[14] M. Spong, "On the force control problem for flexible joint robots," IEEE Trans. Automat. Control, vol. 34, no. 1, pp. 107-111, Jan. 1989.

[15] A. Albu-Schäffer and G. Hirzinger, "A globally stable state-feedback controller for flexible joint robots," J. Adv. Robot., vol. 15, no. 8, pp. 799 814, 2001.

[16] A. Albu-Schäffer, "Regelung von robotern mit elastischen gelenken am beispiel der DLR-leichtbauarme" Ph.D. dissertation, Technische Universität München, München, Germany, 2001.

[17] L. Zollo, B. Siciliano, A. D. Luca, E. Guglielmelli, and P. Dario, "Compliance control for an anthropomorphic robot with elastic joints: Theory and experiments," ASME J. Dyn. Syst., Meas., Control, vol. 127, no. 3, pp. 321-328, 2005 .

[18] A. De Luca, B. Siciliano, and L. Zollo, "Pd control with on-line gravity compensation for robots with elastic joints: Theory and experiments," Automatica, vol. 41, no. 10, pp. 1809-1819, 2005.

[19] G. Hirzinger, A. Albu-Schäffer, M. Hähnle, I. Schaefer, and N. Sporer, "On a new generation of torque controlled light-weight robots," in Proc. IEEE Int. Conf. Robot. Autom., 2001, pp. 3356-3363.

[20] G. Hirzinger, N. Sporer, A. Albu-Schäffer, M. Hähnle, R. Krenn, A. Pascucci, and M. Schedl, "DLR's torque-controlled light weight robot III-Are we reaching the technological limits now?," in Proc. IEEE Int. Conf. Robot. Autom., 2002, pp. 1710-1716.

[21] A. De Luca and P. Tomei, "Elastic joints," in Theory of Robot Control. London, U.K.: Springer-Verlag, 1996, pp. 179-218.

[22] Ch. Ott, "Cartesian impedance control of flexible joint manipulators" Ph.D. dissertation, Saarland University, Saarbrücken, Germany, Nov. 2005. 
[23] Ch. Ott, A. Albu-Schäffer, A. Kugi, S. Stramigioli, and G. Hirzinger, "A passivity based cartesian impedance controller for flexible joint robotsPart I: Torque feedback and gravity compensation," in Proc. IEEE Int. Conf. Robot. Autom., 2004, pp. 2659-2665.

[24] A. Albu-Schäffer, Ch. Ott, and G. Hirzinger, "A passivity based cartesian impedance controller for flexible joint robots-Part II: Full state feedback, impedance design and experiments," in Proc. IEEE Int. Conf. Robot. Autom., 2004, pp. 2666-2672.

[25] Vidyasagar, Nonlinear Systems Analysis, 2nd ed. Englewood Cliffs, NJ: Prentice-Hall, 1993.

[26] A. van der Schaft, $L_{2}$-Gain and Passivity Techniques in Nonlinear Control, 2nd ed. New York: Springer-Verlag, 2000.

[27] A. Kugi, Non-linear Control Based on Physical Models. New York: Springer-Verlag, 2001

[28] S. Zhang and E. D. Fasse, "Spatial compliance modeling using a quaternion-based potential function method," Multibody Syst.Dyn., vol. 4, pp. 75-101, 2000.

[29] C. Natale, Interaction Control of Robot Manipulators: Six-Degreesof-Freedom Tasks. [Springer Tracts in Advanced Robotics (STAR)], vol. 3, New York: Springer-Verlag, 2003.

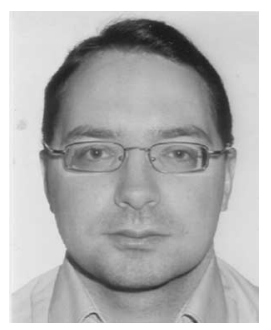

Christian Ott (M’08) received the Dipl.Ing. degree in mechatronics from the Johannes Kepler University (JKU), Linz, Austria, in 2001, and the Dr.-Ing. degree in control engineering from Saarland University, Saarbrücken, Germany, in 2005.

From March 2001 to April 2007, he was with the German Aerospace Center (DLR e.V.), Institute of Robotics and Mechatronics, Wessling, Germany. Since May 2007, he has been a Project Assistant Professor in the Department of Mechano-Informatics, University of Tokyo, Tokyo, Japan. His current research interests include nonlinear control of robotic systems, flexible joint robots, impedance control, and two-armed (humanoid) manipulation.

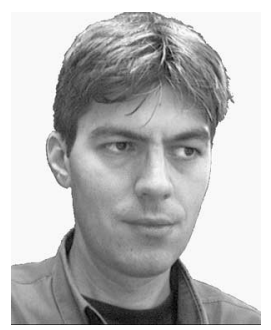

Alin Albu-Schäffer (M'07) received the Diploma degree in electrical engineering from the Technical University of Timisoara, Timisoara, Romania, in 1993, and the Ph.D. degree in control systems from the Technical University of Munich, Munich, Germany, in 2002.

Since 1995, he has been with the German Aerospace Center (DLR), Institute of Robotics and Mechatronics, Wessling, Germany, where he is currently coordinating control and advanced manipulation activities. His current research interests include robot modeling and control, nonlinear control, flexible joint robots, impedance and force control, and physical human-robot interaction.

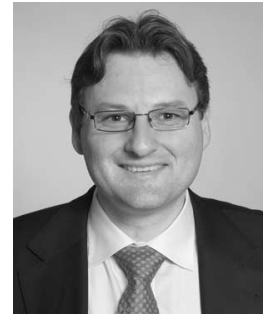

Andreas Kugi (M'94) received the Dipl.Ing. degree in electrical engineering from the Graz University of Technology, Graz, Austria, in 1992, and the Dr.techn. degree in control engineering and the Habilitation degree in automatic control and control theory from the Johannes Kepler University (JKU), Linz, Austria, in 1995 and 2000.

From 1995 to 2000, he was an Assistant Professor at the JKU, where from 2000 to 2002, he was an Associate Professor. In 2002, he was a Full Professor at Saarland University, Saarbrücken, Germany, where he held the Chair of System Theory and Automatic Control until May 2007. Since June 2007, he has been a Full Professor of complex dynamical systems and the Head of the Automation and Control Institute, Vienna University of Technology, Vienna, Austria. His current research interests include the physics-based modeling and control of (nonlinear) mechatronic systems, differential geometric and algebraic methods for nonlinear control, and the controller design for infinite-dimensional systems. He is involved in several industrial research projects in the field of automotive applications, hydraulic servo-drives, smart structures, and rolling mill applications. He is an Associate Editor of the Mechatronics and the Debuty-Editor-in Chief of the Control Engineering Practice.

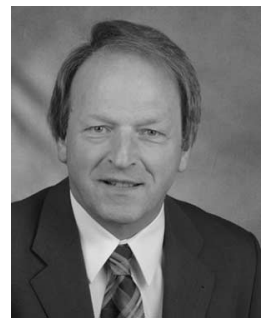

Gerd Hirzinger (F'02) received the Dipl.Ing. and $\mathrm{Ph} . \mathrm{D}$. degrees from the Technical University of $\mathrm{Mu}-$ nich, Munich, Germany, in 1969 and 1974, respectively.

Since 1992, he has been the Director at the the German Aerospace Center (DLR), Institute of Robotics and Mechatronics, Wessling, Germany. In 1991, he received a joint professorship from the Technical University of Munich, and in 2003, an honorary professorship from the Harbin Institute of Technology, China. He was a prime investigator of the first remotely controlled space robot ROTEX, which flew onboard shuttle Columbia in April 1993.

Dr. Hirzinger is the recipient of numerous national and international awards, including the Joseph-Engelberger-Award for achievements in robotic science in 1994, the Leibniz-Award, the highest scientific award in Germany in 1995, the Japan Robotics Association (JARA) Award, the Karl-Heinz-Beckurts-Award, Germany's most important award for outstanding promotion of the partnership between science and industry in 1996, and the IEEE-Fellow Award in 1997. In 2004, he got the order of merit of the Federal Republic of Germany and became member of the "Wall of Fame" of the Heinz Nixdorf Computer Museum. He is also the recipient of the IEEE Pioneer Award of the Robotics and Automation Society and the "Honorary Citizenship" of the Budapest Tech in 2005, and the IEEE Field Award "Robotics and Automation" in 2007. 